\title{
Preparation of Electrode Materials Based on Carbon Cloth via Hydrothermal Method and Their Application in Supercapacitors
}

\author{
Xiaonan Wang ${ }^{1}$, Peiquan $\mathrm{Xu}{ }^{1,2, * \mathbb{D}}$, Pengyu Zhang ${ }^{1}$ and Shuyue Ma ${ }^{1}$ \\ 1 College of Materials Engineering, Shanghai University of Engineering Science, Shanghai 201620, China; \\ 15738773508@163.com (X.W.); pengyu9711@163.com (P.Z.); mashuyue1998@163.com (S.M.) \\ 2 Shanghai Collaborative Innovation Center of Laser Advanced Manufacturing Technology, \\ Shanghai 201620, China \\ * Correspondence: pqxu@sues.edu.cn; Tel.: +86-21-6779-1204
}

Citation: Wang, X.; Xu, P.; Zhang, P.; $\mathrm{Ma}$, S. Preparation of Electrode Materials Based on Carbon Cloth via Hydrothermal Method and Their Application in Supercapacitors. Materials 2021, 14, 7148. https:// doi.org/10.3390/ma14237148

Academic Editors: Dimitra Vernardou and Pedro Gómez-Romero

Received: 20 October 2021

Accepted: 20 November 2021

Published: 24 November 2021

Publisher's Note: MDPI stays neutral with regard to jurisdictional claims in published maps and institutional affiliations.

Copyright: (c) 2021 by the authors. Licensee MDPI, Basel, Switzerland. This article is an open access article distributed under the terms and conditions of the Creative Commons Attribution (CC BY) license (https:// creativecommons.org/licenses/by/ $4.0 /)$.

\begin{abstract}
Supercapacitors have the unique advantages of high power density, fast charge and discharge rates, long cycle life, high safety, and reliability, and are increasingly being used for applications including automobiles, rail transit, communication equipment, digital electronics, and aerospace equipment. The supercapacitor industry is currently in a stage of rapid development; great breakthroughs have also been made in improving the performance of supercapacitors and the expansion of their application. Electrode technology is the core of supercapacitors. Transition-metal compounds have a relatively high theoretical capacity and have received widespread attention as electrode materials for supercapacitors. In addition, there is a synergistic effect between the different components of various electrode composite materials. Due to their superior electrochemical performance, supercapacitors are receiving increasing research attention. Flexible supercapacitors have been hailed for their good plasticity, resulting in a development boom. This review article mainly outlines the development process of various electrode materials, including carbon materials, conductive polymers, metal compounds, and composite materials, as well as flexible electrode materials based on carbon cloth.
\end{abstract}

Keywords: supercapacitors; carbon materials; conductive polymers; metal compounds; composite materials; carbon cloth

\section{Introduction}

Recently, the explosive growth of society and constant advancement of science and technology have led to an increasing demand for energy, resulting in a significant energy crisis and environmental problems [1,2]. There is thus an urgent need to develop environmentally friendly, efficient, clean, and sustainable advanced energy conversion and storage technologies $[3,4]$. Currently, the development of the new energy industry is based on the three core products of secondary batteries (mainly lithium-ion batteries) [5], supercapacitors [6], and fuel cells [7], with a vast application network.

Supercapacitors are also known as large-capacity capacitors, energy storage capacitors, gold capacitors, electric double layer capacitors, and farad capacitors. After decades of development, using carbon material from naturally abundant lignocellulose biomass precursors as the electrode material of flexible/wearable electronic devices is a future trend, but there are still technological challenges [8]. Based on availability and low-cost, various carbon materials, such as activated carbons (AC), nitrogen doping on carbon, and graphene on carbon cloth, have also been studied $[9,10]$. However, their performance is not particularly good.

Initially, in 1957, Becker filed an application to use high-specific-surface-area activated carbon as the electrode material for supercapacitors. In 1962, the Standard Oil Company 
commercialized carbon material electrochemical capacitors. In 1979, the Nippon Electronic Company (NEC) implemented supercapacitors for large-scale commercial applications. Scholars are increasingly discovering the application value of supercapacitors. The structure of a supercapacitor consists of the electrodes, electrolyte, and separator. The electrode material in the electrodes plays a vital role in the performance of the supercapacitor [11]. The electrode material is the core. From carbon materials [12,13], element-doped carbon materials [14,15], metal compounds [16,17], multi-metal compounds [18,19], and polymers $[20,21]$, to various composite materials, various superior porous three-dimensionally structured composite materials have been constructed. With the further development of society, there has been a greater pursuit of wearable and strong plasticity flexible electrodes. The preparation of carbon cloth offers the advantages of low cost, good flexibility, and good conductivity, and, as a substrate, has been hailed as a high-performance electrode material [22-26].

CC has been used as an electrode material for flexible supercapacitors applied in portable and wearable products, setting off a research boom. Figure 1 clearly shows that in the past ten years, there has been a linear upward trend in the number of publications with the keywords "supercapacitor", "carbon cloth", and "carbon fiber cloth" included in their titles (from a search in Web of Science), and this trend is expected to be maintained. Flexible supercapacitors based on CC are very promising electrode materials. CC has the advantages of light weight, low cost, excellent flexibility, large surface area, high conductivity, and porosity; in addition, it is particularly easy to construct an electrode material with structural advantages.

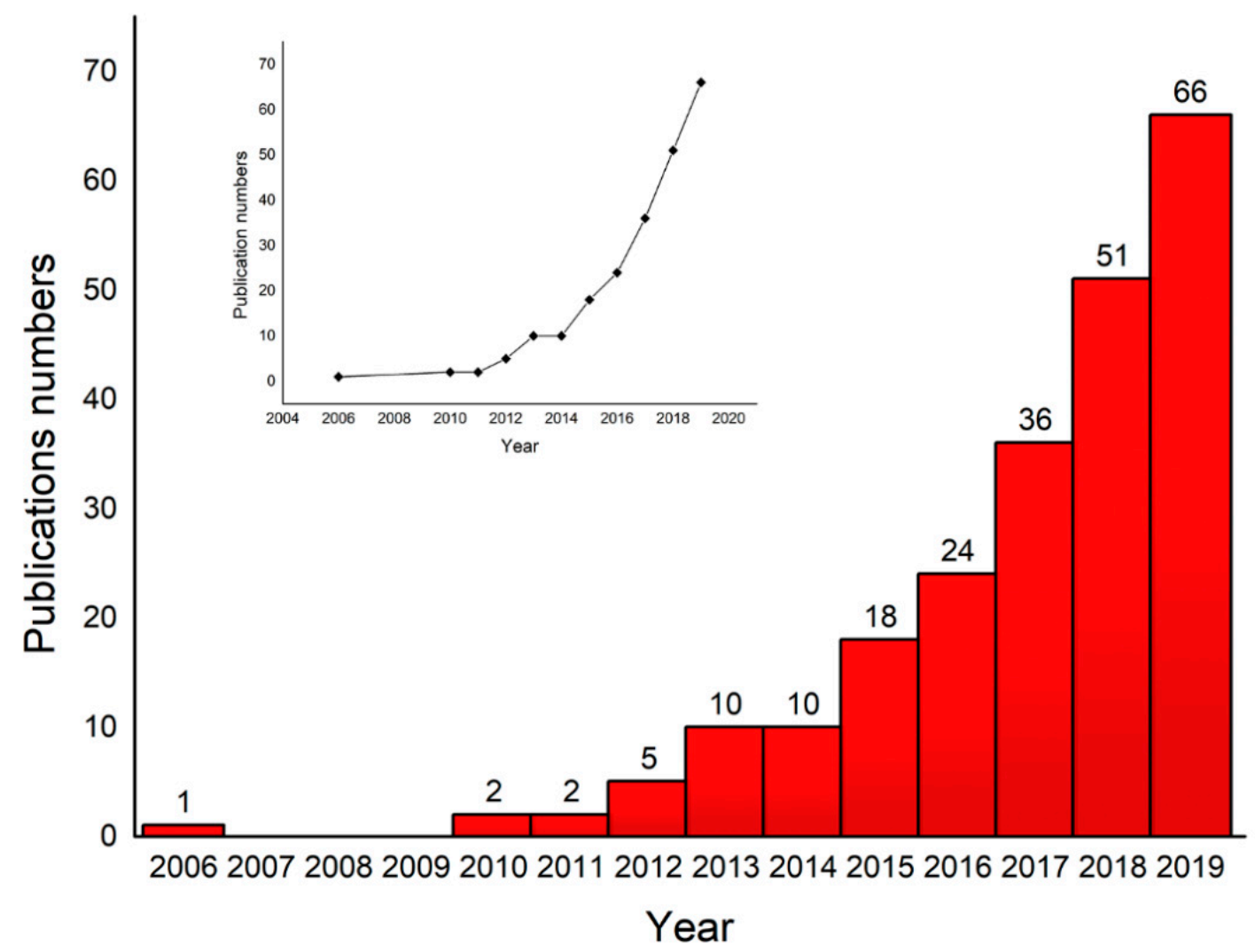

Figure 1. Increasing trend of the number of publications related to supercapacitors based on CC in the past decade. These results were obtained by searching the Web of Science for articles with the keywords "supercapacitor", "carbon cloth", and "carbon fiber cloth" included in their titles. 


\section{Electrode Material}

\subsection{Carbon Materials}

Carbon materials are mainly used as the electrode materials for Type I supercapacitors and were the earliest electrode materials to be researched and applied. As shown in Table 1, Gamby et al. [27] conducted the first electrochemical characteristic tests on various activated carbons from the PICA Company and observed that the highest specific capacitance was $125 \mathrm{~F} \cdot \mathrm{g}^{-1}$. Beck et al. [28] prepared industrial carbon black (CB) electrodes with polytetrafluoroethylene (PTFE) as a binder in $12 \mathrm{M} \mathrm{H}_{2} \mathrm{SO}_{4}$; the highest capacitance measured was $250 \mathrm{~F} \cdot \mathrm{g}^{-1}$. Carbon aerogel is a lightweight porous carbon material with a three-dimensional nanonetwork structure. It has a large specific surface area, low density, high porosity, adjustable pore size distribution, and good electrical conductivity. Fischer et al. [29] obtained carbon aerogel with a density of $800 \mathrm{~kg} \cdot \mathrm{cm}^{-3}$ via pyrolysis from resorcinol formaldehyde aerogel; the highest capacity measured was $46 \mathrm{~F} \cdot \mathrm{cm}^{-3}$. Li et al. [30] synthesized mixed carbon aerogels by blending cresol, catechol, and formaldehyde, with a high specific capacitance of $77 \mathrm{~F} \cdot \mathrm{cm}^{-3}\left(104 \mathrm{~F} \cdot \mathrm{g}^{-1}\right)$. The good porous structure of carbon aerogels results in their high specific capacitance. Carbon fiber is an inorganic polymer fiber with a carbon content higher than $90 \%$. It has the characteristics of low density, light weight, high strength, and high elastic modulus.

Carbon nanotubes (CNTs) appear as a seamless centrally controlled nanoscale coaxial cylinder rolled by sheet-structured graphite, with fullerene hemispheres sealed at both ends, divided into single-walled carbon nanotubes (SWCNTs) and multi-walled carbon nanotubes (MWCNTs). Compared with the best activated carbon, MWCNTs have available mesoporous structure and the value of their specific capacitance varies up to $135 \mathrm{~F} \cdot \mathrm{g}^{-1}$ [31] Liu et al. [32] deposited CNTs on a platinum electrode for the first time, resulting in a specific capacitance of $283 \mathrm{~F} \cdot \mathrm{g}^{-1}$, which was twice that of an active carbon electrode under the same conditions. Pan et al. [33] reported a tube-to-tube multi-walled carbon nanotube with a specific capacitance of $135 \mathrm{~F} \cdot \mathrm{g}^{-1}$ consisting of outer nanotubes with an average outer diameter of $50 \mathrm{~nm}$ and internal nanotubes with diameters of 3-10 nm. Yang et al. [34] introduced hierarchical graphene sheet (GS)-CNTs with a specific capacitance of $326.5 \mathrm{~F} \cdot \mathrm{g}^{-1}$. The stacking of GS can be efficaciously impeded by inserting an appropriate quantity of CNTs as nanospacers and enlarging the space between GS sheets, leading to a highly porous nanostructure.

Because of the unique porous structure of carbon materials, they have a larger specific surface area, which facilitates the mass diffusion and transport of electrolytes, providing more ion contact sites and increasing the capacitance. Hollow core, mesoporous shell carbon nanospheres (HCMSs) with a high surface area of $1704 \mathrm{~m}^{2} \cdot \mathrm{g}^{-1}$ exhibited a high specific capacity of $251 \mathrm{~F} \cdot \mathrm{g}^{-1}$ at $50 \mathrm{mV} \cdot \mathrm{s}^{-1}$ in $2 \mathrm{M} \mathrm{H}_{2} \mathrm{SO}_{4}$ [35]. Onion-like carbon (OLC) [36] and nonporous carbons (NPCs) with a metal-organic framework (MOF) as a template exhibited excellent mesoporous characteristics [37]. Zhao et al. [38] fabricated sulfurcontaining mesoporous carbons via one-pot aqueous self-assembly with different chemical states of sulfur in tunable amounts. The addition of inorganic elements such as $\mathrm{O}, \mathrm{N}, \mathrm{S}$, and $\mathrm{P}$ improved the electrochemical performance.

Table 1. Carbon materials for supercapacitors.

\begin{tabular}{|c|c|c|c|c|c|c|}
\hline Material & $\begin{array}{l}\text { The Highest } \\
\text { Capacities }\end{array}$ & $\begin{array}{c}\text { Specific } \\
\text { Surface Area }\end{array}$ & $\begin{array}{l}\text { Specific Power } \\
\text { Densities }\end{array}$ & $\begin{array}{l}\text { Specific Energy } \\
\text { Densities }\end{array}$ & $\begin{array}{c}\text { Specific Capacitance } \\
\text { Retention }\end{array}$ & Ref. \\
\hline $\begin{array}{l}\text { Activated } \\
\text { carbon }\end{array}$ & $125 \mathrm{~F} \cdot \mathrm{g}^{-1}$ & - & - & - & - & [27] \\
\hline CBs & $250 \mathrm{~F} \cdot \mathrm{g}^{-1}$ & $28-1690 \mathrm{~m}^{2} \cdot \mathrm{g}^{-1}$ & - & - & - & [28] \\
\hline $\begin{array}{l}\text { Carbon } \\
\text { aerogels }\end{array}$ & $46 \mathrm{~F} \cdot \mathrm{cm}^{-3}$ & 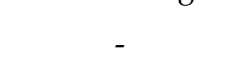 & - & - & - & [29] \\
\hline
\end{tabular}


Table 1. Cont.

\begin{tabular}{|c|c|c|c|c|c|c|}
\hline Material & $\begin{array}{l}\text { The Highest } \\
\text { Capacities }\end{array}$ & $\begin{array}{c}\text { Specific } \\
\text { Surface Area }\end{array}$ & $\begin{array}{l}\text { Specific Power } \\
\text { Densities }\end{array}$ & $\begin{array}{l}\text { Specific Energy } \\
\text { Densities }\end{array}$ & $\begin{array}{c}\text { Specific Capacitance } \\
\text { Retention }\end{array}$ & Ref. \\
\hline $\begin{array}{l}\text { Carbon } \\
\text { aerogels }\end{array}$ & $\begin{array}{l}77 \mathrm{~F} \cdot \mathrm{cm}^{-3} \\
\left(104 \mathrm{~F} \cdot \mathrm{g}^{-1}\right)\end{array}$ & - & - & - & - & [30] \\
\hline MWCNTs & $135 \mathrm{~F} \cdot \mathrm{g}^{-1}$ & $470 \mathrm{~m}^{2} \cdot \mathrm{g}^{-1}$ & - & - & - & [31] \\
\hline SWCNTs & $283 \mathrm{~F} \cdot \mathrm{g}^{-1}$ & - & - & - & - & [32] \\
\hline $\begin{array}{c}\text { Tubes-in-tube } \\
\text { CNTs }\end{array}$ & $315 \mathrm{~F} \cdot \mathrm{g}^{-1}$ & - & 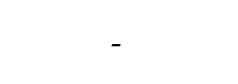 & 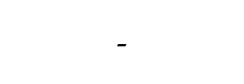 & - & [33] \\
\hline GS-CNTs & $326.5 \mathrm{~F} \cdot \mathrm{g}^{-1}$ & - & $78.29 \mathrm{~kW} \cdot \mathrm{kg}^{-1}$ & $21.74 \mathrm{Wh} \cdot \mathrm{kg}^{-1}$ & - & [34] \\
\hline HCMSs & $251 \mathrm{~F} \cdot \mathrm{g}^{-1}$ & - & - & - & 98\% (after 200 cycles) & [35] \\
\hline OLC & - & - & $195.0 \mathrm{~W} \cdot \mathrm{cm}^{-3}$ & $2.9 \mathrm{mWh} \cdot \mathrm{cm}^{-3}$ & - & [36] \\
\hline NPCs & $222 \mathrm{~F} \cdot \mathrm{g}^{-1}$ & - & - & - & - & [37] \\
\hline S-carbon & - & - & - & - & - & [38] \\
\hline
\end{tabular}

\subsection{Conductive Polymers}

Conductive polymers have caused a research boom. Shirakawa et al. [39] first prepared iodine-doped polyacetylene with a conductivity of up to $10^{3} \mathrm{~S} \cdot \mathrm{cm}^{-1}$. Conductive polymers have the characteristics of high electrical conductivity, large specific surface area, and light specific gravity, as well as good flexibility, low production cost, and high energy efficiency; therefore, they can be used for rechargeable and secondary batteries and electrode materials [40]. Arbizzian et al. [41] synthesized three supercapacitors: a symmetric supercapacitor based on p-doped poly(pyrrole), an asymmetric supercapacitor based on both p-doped poly(pyrrole) and poly(3-methylthiophene), and a symmetric supercapacitor based on $\mathrm{p}$ - and n-doped poly(dithieno[3,4-b:3 $\left.3^{\prime}, 4^{\prime}-\mathrm{d}\right]$ thiophene) with a high working potential. In Table 2, Ferraris et al. [42] studied various poly 3-(phenylthiophene) derivatives as electrode materials to assemble supercapacitors. The capacitor based on 3-(3, 4 difluorophenyl) thiophene (MPFPT) was particularly promising because of its high capacity and excellent charge/discharge performance. Fusalba et al. [43] synthesized poly(cyclopenta [2,1-b;3,4-b'] dithiophen-4-one) (PCDT) with an open and porous structure. For both the p-type and n-type doped states, the material exhibited a low-frequency capacitance of approximately $70 \mathrm{~F} \cdot \mathrm{g}^{-1}$.

However, conductive polymers also have shortcomings, such as poor mechanical properties and long-term cycle stability. To overcome these shortcomings and enhance their performance, the optimization of the structure and shape or the mixture of conductive polymers with other carbon materials are important routes. Gouérec et al. [44] showed that the high performance of polyacrylonitrile (PAN) microcellular foam thin films deposited on carbon fibers is related to their high surface area and micropore distribution. Pyrrole (Py) on the surface of a porous graphite fiber matrix resulted in a specific capacitance of $400 \mathrm{~F} \cdot \mathrm{g}^{-1}$ [45]. Polypyrrole (PPy) on MWCNT membranes exhibits high redox activity, resulting in high specific capacitance [46]. Dubal et al. [47] observed that multilayer PPy nanosheets exhibited a specific capacitance of $586 \mathrm{~F} \cdot \mathrm{g}^{-1}$ at a scan rate of $2 \mathrm{mV} \cdot \mathrm{s}^{-1}$, with a Brunauer-Emmett-Teller (BET) surface area of $37.1 \mathrm{~m}^{2} \cdot \mathrm{g}^{-1}$, which is higher than that of PPy nanoribbons and nanobricks. Jyothibasu et al. [48] prepared PPy tubes using onestep in situ chemical oxidative polymerization with curcumin as a template, which was combined with functionalized carbon nanotubes (f-CNTs) as electrode materials. The electrodes exhibited morphological uniformity, a favorable hierarchical porous structure, a large surface area, and excellent electrochemical properties, including outstanding cycling stability (retention of $118.18 \%$ of the initial capacitance after 12,500 charge/discharge cycles). 
Table 2. Properties of conductive polymers for supercapacitors prepared by various methods.

\begin{tabular}{|c|c|c|c|c|c|}
\hline Materials & Method & $\begin{array}{c}\text { Specific } \\
\text { Capacitance }\end{array}$ & $\begin{array}{l}\text { Specific Power } \\
\text { Densities }\end{array}$ & $\begin{array}{l}\text { Specific Energy } \\
\text { Densities }\end{array}$ & Ref. \\
\hline $\begin{array}{c}\text { Poly 3- } \\
\text { (Phenylthiophene) } \\
\text { derivatives }\end{array}$ & $\begin{array}{l}\text { Electrochemical } \\
\text { polymerization }\end{array}$ & - & $5 \mathrm{~kW} \cdot \mathrm{kg}^{-1}$ & $50 \mathrm{Wh} \cdot \mathrm{kg}^{-1}$ & [42] \\
\hline PCDT & $\begin{array}{l}\text { Electrochemical } \\
\text { polymerization }\end{array}$ & - & $1 \mathrm{~kW} \cdot \mathrm{kg}^{-1}$ & $6 \mathrm{Wh} \cdot \mathrm{kg}^{-1}$ & [43] \\
\hline PAN & Deposition & - & - & - & [44] \\
\hline Py & $\begin{array}{c}\text { Chemical } \\
\text { polymerization }\end{array}$ & $400 \mathrm{~F} \cdot \mathrm{g}^{-1}$ & - & - & [45] \\
\hline PPy & $\begin{array}{l}\text { Electrochemical } \\
\text { deposition }\end{array}$ & $427 \mathrm{~F} \cdot \mathrm{g}^{-1}$ & - & - & [46] \\
\hline PPy & $\begin{array}{c}\text { Electro } \\
\text { polymerization }\end{array}$ & $586 \mathrm{~F} \cdot \mathrm{g}^{-1}$ & - & - & [47] \\
\hline PPy & $\begin{array}{l}\text { In-situ chemical } \\
\text { oxidative } \\
\text { polymerization }\end{array}$ & $2732 \mathrm{mF} \cdot \mathrm{cm}^{-2}$ & $129.35 \mathrm{~mW} \cdot \mathrm{cm}^{-2}$ & $242.84 \mu \mathrm{Wh} \cdot \mathrm{cm}^{-2}$ & [48] \\
\hline $\begin{array}{l}(\mathrm{E})-\alpha- \\
\text { cyanoethylene } \\
\text { thiophene } \\
\text { derivatives }\end{array}$ & $\begin{array}{l}\text { Electrochemical } \\
\text { polymerization }\end{array}$ & - & $1.6 \mathrm{~kW} \cdot \mathrm{kg}^{-1}$ & $8.6 \mathrm{Wh} \cdot \mathrm{kg}^{-1}$ & [49] \\
\hline
\end{tabular}

\subsection{Metal Compounds}

As electrode materials, metal compounds exhibit excellent pseudocapacitance behavior (superior to that of carbon materials), owing to their rapid reversible redox reactions. $\mathrm{RuO}_{2}$ is the earliest metal compound used as an electrode material for supercapacitors. Amorphous $\mathrm{RuO}_{2} \cdot \mathrm{xH}_{2} \mathrm{O}$ is conducive to the diffusion of electrolyte ions in its body phase. The redox reaction not only occurs on the surface of the electrode, but also inside, which is helpful for improving the utilization rate and achieving a high specific capacitance. Compared with $\mathrm{RuO}_{2} \cdot \mathrm{xH}_{2} \mathrm{O}$ powder $[50,51]$ (Table 3), $\mathrm{RuO}_{2} \cdot \mathrm{xH}_{2} \mathrm{O}$ thin film is more uniform and complete and has a higher specific capacitance. Scholars have also discovered that the introduction of other metals is helpful for increasing the capacitance because of the synergistic effect between the metals [52,53]. However, the poor conductivity, high cost, and toxicity of $\mathrm{RuO}_{2}$ prevents its use in large-scale commercial applications. At this time, relatively inexpensive transition-metal compounds were also shown to possess good pseudocapacitor performance, which was widely valued.

Conway [54] proposed that inexpensive transition-metal oxides have pseudocapacitive behavior, and they were considered the most promising energy storage materials. Prasad et al. [55] prepared nanorod-shaped three-dimensional $\operatorname{In}_{2} \mathrm{O}_{3}$ with high specific capacitance and high power density that exhibited good electrical performance similar to that of the expensive $\mathrm{RuO}_{2}$. Nam et al. [56] reported the largest specific capacitance of $\mathrm{NiO}$ obtained by electrochemical precipitation with heat treatment at $300{ }^{\circ} \mathrm{C}$; this material has defect properties that can improve its electrochemical properties. In addition to transitionmetal oxides [57-61], sulfides, phosphides, and selenides also exhibit pseudocapacitive behavior. These elements' atoms have larger ionic radii and are more prone to transition transfer and discrete diffusion. To some extent, these materials exhibit improved electrochemical performance. Sulfur atoms have a lower electronegativity than oxygen atoms, and sulfides have a faster redox reaction rate and higher electronic conductivity [62]. Qian et al. [63] prepared CuS nanotubes with a high specific capacitance of $2393 \mathrm{~F} \cdot \mathrm{g}^{-1}$ at a scan rate of $10 \mathrm{mV} \cdot \mathrm{s}^{-1}$. This was the first time that a novel redox-active alkaline electrolyte (polysulfide electrolyte) was used to improve the specific capacitance. A multilayer M$\mathrm{MoS}_{2}-\mathrm{H}_{2} \mathrm{O}$ system was first investigated; nanochannels between layers with a distance of approximately $1.18 \mathrm{~nm}$ increased the space for ion diffusion and extended the surface area for adsorption [64]. 
Nano-size compounds with high specific surface area have more active sites and can fully contact the electrolyte, which is beneficial for increasing the specific capacitance [65-70]. For the first time, Ramasamy et al. [71] developed a simple colloidal method for the synthesis of $\mathrm{CuSbSe}_{x} \mathrm{~S}_{2-x}$ by replacing $\mathrm{S}$ with Se; the materials exhibited excellent cyclic stability with promising specific capacitance values. Se atoms can be used to adjust the width of the interlayer gap between layers, which is beneficial for ion diffusion. Lin et al. [72] synthesized coral-like $\mathrm{LiFePO}_{4}$ particles through a facile chemical etching method with rugby-like $\mathrm{LiFePO}_{4}$ particles as precursors. Priyadharsin et al. [73] prepared $\gamma-\mathrm{KCoPO}_{4}$ nanocrystals as supercapacitor electrodes in an aqueous electrolyte for the first time, resulting in a specific capacitance of $309 \mathrm{C} \cdot \mathrm{g}^{-1}$ at $1 \mathrm{mV} \cdot \mathrm{s}^{-1}$ in $1 \mathrm{M} \mathrm{KOH}$. Multi-metal compounds exhibit improved electrochemical performance because, in addition to the synergistic pictograms between different components, it is also easy to construct various morphologies with structural advantages [68-70,72,74,75].

In general, transition metals are low in cost, easy to obtain, abundant, and exhibit excellent pseudocapacitance performance. Combining transition metals with other materials to construct porous three-dimensional nanostructures with high specific surface area results in electrode materials with high specific capacitance, good energy and power density, and good stability.

Table 3. Properties of metal compounds for supercapacitors prepared by various methods.

\begin{tabular}{|c|c|c|c|c|c|c|}
\hline Materials & Method & $\begin{array}{c}\text { Specific } \\
\text { Capacitance }\end{array}$ & $\begin{array}{l}\text { Specific Power } \\
\text { Density }\end{array}$ & $\begin{array}{c}\text { Specific Energy } \\
\text { Density }\end{array}$ & $\begin{array}{c}\text { Specific Capacitance } \\
\text { Retention }\end{array}$ & Ref. \\
\hline $\begin{array}{c}\mathrm{RuO}_{2} \cdot \mathrm{xH}_{2} \mathrm{O} \\
\text { powder }\end{array}$ & Sol-gel process & $720 \mathrm{~F} \cdot \mathrm{g}^{-1}$ & - & $26.7 \mathrm{Wh} \cdot \mathrm{kg}^{-1}$ & - & [50] \\
\hline $\mathrm{RuO}_{2}$ films & Electrodeposition & $1190 \mathrm{~F} \cdot \mathrm{g}^{-1}$ & - & - & - & [51] \\
\hline $\mathrm{Ru}_{0.6} \mathrm{Sn}_{0.4} \mathrm{O}_{2} \cdot \mathrm{nH}_{2} \mathrm{O}$ & Hydrothermal & $830 \mathrm{~F} \cdot \mathrm{g}^{-1}$ & - & - & - & [53] \\
\hline $\begin{array}{l}\mathrm{RuO}_{2}-\mathrm{VO}_{2} \text { solid } \\
\text { solution }\end{array}$ & $\begin{array}{l}\text { Polymerizable- } \\
\text { complex }\end{array}$ & $1210 \mathrm{~F} \cdot \mathrm{g}^{-1}$ & - & - & - & [54] \\
\hline $\mathrm{In}_{2} \mathrm{O}_{3}$ & $\begin{array}{l}\text { Electrochemical } \\
\text { deposition }\end{array}$ & $190 \mathrm{~F} \cdot \mathrm{g}^{-1}$ & - & - & - & [55] \\
\hline $\mathrm{NiO}$ film & $\begin{array}{l}\text { Electrochemical } \\
\text { precipitation }\end{array}$ & - & - & - & - & [56] \\
\hline $\mathrm{NiO}$ nanosheet & Hydrothermal & $989 \mathrm{Fg}-1$ & - & $49.45 \mathrm{Wh} \cdot \mathrm{kg}^{-1}$ & $\begin{array}{c}97 \% \text { (after } 1000 \\
\text { cycles) }\end{array}$ & [57] \\
\hline cobalt oxide & Deposition & $165 \mathrm{~F} \cdot \mathrm{g}^{-1}$ & - & - & - & [58] \\
\hline $\begin{array}{l}\mathrm{CuO} \text { multilayer } \\
\text { nanosheets }\end{array}$ & CBD & $43 \mathrm{~F} \cdot \mathrm{g}^{-1}$ & - & - & - & [59] \\
\hline $\mathrm{MnO}_{2}$ & - & $398 \mathrm{~F} \cdot \mathrm{g}^{-1}$ & - & - & - & [60] \\
\hline $\mathrm{Mn}_{3} \mathrm{O}_{4}$ thin films & SILAR & $314 \mathrm{~F} \cdot \mathrm{g}^{-1}$ & - & - & - & [61] \\
\hline CoS nanowires & $\begin{array}{l}\text { Biomolecule- } \\
\text { assisted } \\
\text { hydrothermal }\end{array}$ & $508 \mathrm{~F} \cdot \mathrm{g}^{-1}$ & - & - & - & [62] \\
\hline CuS nanotubes & - & $2393 \mathrm{~F} \cdot \mathrm{g}^{-1}$ & - & - & - & [63] \\
\hline $\begin{array}{c}\mathrm{M}-\mathrm{MoS}_{2}-\mathrm{H}_{2} \mathrm{O} \\
\text { system }\end{array}$ & Hydrothermal & $380 \mathrm{~F} \cdot \mathrm{g}^{-1}$ & - & - & - & [64] \\
\hline$\frac{\mathrm{Ni}(\mathrm{OH})_{2}}{\alpha-\mathrm{Co}(\mathrm{OH})_{2}}$ & Hydrothermal & $1715 \mathrm{~F} \cdot \mathrm{g}^{-1}$ & - & - & - & [65] \\
\hline $\begin{array}{l}\text { nanowire arrays } \\
\text { (NWAs) }\end{array}$ & Hydrothermal & $642.5 \mathrm{~F} \cdot \mathrm{g}^{-1}$ & - & - & - & [66] \\
\hline $\begin{array}{c}\mathrm{FeVO}_{4} \\
\text { nanoparticles }\end{array}$ & $\begin{array}{c}\text { Co- } \\
\text { precipitation }\end{array}$ & $972 \mathrm{~F} \cdot \mathrm{g}^{-1}$ & $1326 \mathrm{~kW} \cdot \mathrm{kg}^{-1}$ & $21 \mathrm{Wh} \cdot \mathrm{kg}^{-1}$ & - & [67] \\
\hline $\begin{array}{c}\mathrm{NiCO}_{2} \mathrm{O}_{4} \\
\text { NSs@HMRAs }\end{array}$ & Electrodeposition & $678 \mathrm{~F} \cdot \mathrm{g}^{-1}$ & - & $15.42 \mathrm{Wh} \cdot \mathrm{kg}^{-1}$ & $\begin{array}{c}96.06 \% \text { (after } 1500 \\
\text { cycles) }\end{array}$ & [68] \\
\hline $\mathrm{CoNi}_{2} \mathrm{~S}_{4}$ & Hydrothermal & $2906 \mathrm{~F} \cdot \mathrm{g}^{-1}$ & $409 \mathrm{~W} \cdot \mathrm{kg}^{-1}$ & $33.9 \mathrm{Wh} \cdot \mathrm{kg}^{-1}$ & - & [69] \\
\hline
\end{tabular}


Table 3. Cont.

\begin{tabular}{|c|c|c|c|c|c|c|}
\hline Materials & Method & $\begin{array}{c}\text { Specific } \\
\text { Capacitance }\end{array}$ & $\begin{array}{l}\text { Specific Power } \\
\text { Density }\end{array}$ & $\begin{array}{c}\text { Specific Energy } \\
\text { Density }\end{array}$ & $\begin{array}{c}\text { Specific Capacitance } \\
\text { Retention }\end{array}$ & Ref. \\
\hline $\begin{array}{l}\text { Sn-Co binary oxide } \\
\text { nanosheets }\end{array}$ & Hydrothermal & $937.4 \mathrm{~F} \cdot \mathrm{g}^{-1}$ & - & - & $\begin{array}{c}97.5 \% \text { (after } 20,000 \\
\text { cycles) }\end{array}$ & [70] \\
\hline $\mathrm{CuSbSe}_{2}$ & Colloidal & - & - & - & $\begin{array}{c}99.5 \% \text { (after } 200 \\
\text { cycles) }\end{array}$ & [71] \\
\hline $\begin{array}{c}\text { Coral-Like } \mathrm{LiFePO}_{4} \\
\text { Particles }\end{array}$ & $\begin{array}{l}\text { Chemical } \\
\text { etching }\end{array}$ & $359 \mathrm{~F} \cdot \mathrm{g}^{-1}$ & - & - & $\begin{array}{c}82.3 \% \text { (after } 2000 \\
\text { cycles) }\end{array}$ & [72] \\
\hline$\gamma-\mathrm{KCoPO} 4$ & Sol-gel & $222 \mathrm{C} \cdot \mathrm{g}^{-1}$ & $1.6 \mathrm{~kW} \cdot \mathrm{kg}^{-1}$ & $28 \mathrm{Wh} \cdot \mathrm{kg}^{-1}$ & - & [73] \\
\hline Mn-Co-Fe HNPs & Electrodeposition & $1200 \mathrm{~F} \cdot \mathrm{g}^{-1}$ & $1125 \mathrm{~W} \cdot \mathrm{kg}^{-1}$ & $11.4 \mathrm{Wh} \cdot \mathrm{kg}^{-1}$ & $\begin{array}{c}96 \% \text { (after } 4000 \\
\text { cycles) }\end{array}$ & [74] \\
\hline $\begin{array}{l}\text { Co-Mo-O-S porous } \\
\text { microspheres }\end{array}$ & Hydrothermal & $1134 \mathrm{~F} \cdot \mathrm{g}^{-1}$ & - & $67.6 \mathrm{Wh} \cdot \mathrm{kg}^{-1}$ & 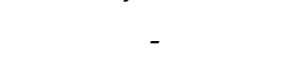 & [75] \\
\hline
\end{tabular}

\subsection{Composite Materials}

Composite materials are more suitable for the development of supercapacitor electrode materials because of their improved electrochemical performance relative to that of single-component materials, resulting from the synergy between the components. It is easier to build porous nanostructures with high specific surface area, which can provide more electroactive sites and enable fast electron transmission and enhanced structural stability. As shown in Table 4, many attempts have been made to study composite materials with carbon materials [76-79]. Electrochemical measurements showed that the pseudocapacitance behavior of $\mathrm{RuO}_{2}$ increases the specific capacitance of pure carbon materials. Transition metals have the potential to replace precious metal oxides in supercapacitor electrodes. Scientists are also committed to developing a low-cost, high-performance material. Composite materials of transition-metal compounds and various carbon materials exhibit better performance than pure carbon materials [80-85]. It is worth emphasizing the use of CNTs, graphene, carbon fiber, and molecular sieves. These materials have high specific surface area and mesoporous structures, which are very conducive to the construction of composite materials with structural advantages [86]. $\mathrm{Co}(\mathrm{OH})_{2} /$ ultrastable $\mathrm{Y}$ molecular sieve composites [87], $\mathrm{NiO} / \mathrm{CNT}$ composites [88], $\mathrm{Co}_{3} \mathrm{O}_{4}$ nanowire/three-dimensional graphene foam [89], $\mathrm{Ni}_{3} \mathrm{~S}_{2}$ nanoparticles/MWCNTs [90], and $\alpha-\mathrm{Ni}(\mathrm{OH})_{2}-\mathrm{GO}$ composites [91] all exhibit high specific capacitance, with the highest capacitance reached being $1760.72 \mathrm{~F} \cdot \mathrm{g}^{-1}$ [91].

Binary and even multi-component metal compound composite materials exhibit superior performance because of the synergistic effect between metals and between different components. $\mathrm{BiMnO}_{5} / \mathrm{MWCNT}$ composites were used as a new active material for positive electrodes of supercapacitors, with a maximum energy density of $9.0 \mathrm{Wh} \cdot \mathrm{kg}^{-1}$ and maximum power density of $2.5 \mathrm{~kW} \cdot \mathrm{kg}^{-1}$ for an asymmetric device [92]. A Co-Al layered double hydroxide nanosheet (Co-Al LDH-NS)/GO film composite [93] exhibited a specific capacitance of $1200 \mathrm{~F} \cdot \mathrm{g}^{-1}$ and a long cyclic life owing to the well-organized layered structure, which was beneficial to efficient electron transport. A $\mathrm{CoMoO}_{4} / \mathrm{G}$ composite exhibited low electrochemical resistance, good rate capability, and good cycle life, and possessed a higher specific surface area and electroactive area than pure $\mathrm{CoMoO}_{4}$, promoting the acquisition of $\mathrm{OH}^{-}$and fast charge transfer [94]. For $\mathrm{N}$-doped graphene, the $\mathrm{N}$ atoms inserted in the carbon lattice played a significant role in improving the capacitance and stability of the electrode material because of the local structural deformation around $\mathrm{N}$ atoms as well as the Coulomb effect $[95,96]$. 
Co-incorporated $\mathrm{NiV}_{2} \mathrm{O}_{6} / \mathrm{Ni}\left(\mathrm{HCO}_{3}\right)$ nanosheet arrays directly grown on $\mathrm{Ni}$ foam possessed long-term durability, with $106.2 \%$ retention of the initial capacity after 10,000 charging/discharging cycles at $100 \mathrm{~mA} \cdot \mathrm{cm}^{-2}$. The electrons transferred from the $\mathrm{V}$ center to the $\mathrm{Ni}$ active sites because of the synergistic contribution of the individual components, making the structure more stable [97]. Composite materials with multiple metal components exhibit high specific capacitance, good rate capability, and improved cycling stability, profiting from the high specific surface area, plentiful surface-active sites, good interfacial conductivity, and porous structure [98-101].

Building composite materials with a well-layered core-shell structure is conducive to enhancing electrochemical performance. Because of the synergistic contribution between the core and shell, the high specific surface area provides more electroactive sites for Faradaic redox reactions and increases ion and electron diffusion; thus, composite materials with core-shell structures exhibit satisfactory electrochemical performance [102-104].

Table 4. Properties of composite materials for supercapacitors prepared by various methods.

\begin{tabular}{|c|c|c|c|c|c|c|}
\hline Materials & Method & $\begin{array}{l}\text { Specific } \\
\text { Capacitance } \\
\text { Density }\end{array}$ & $\begin{array}{l}\text { Specific Power } \\
\text { Density }\end{array}$ & $\begin{array}{c}\text { Specific Energy } \\
\text { Density }\end{array}$ & $\begin{array}{c}\text { Specific } \\
\text { Capacitance } \\
\text { Retention }\end{array}$ & Ref. \\
\hline $\begin{array}{c}\text { Carbon-ruthenium } \\
\text { xerogels }\end{array}$ & Sol-gel & $256 \mathrm{~F} \cdot \mathrm{g}^{-1}$ & - & - & $\begin{array}{r}\text { Almost } 100 \% \\
(>2000 \text { cycles) }\end{array}$ & [76] \\
\hline $\mathrm{RuO}_{2} / \mathrm{CNT}$ & - & $340 \mathrm{~F} \cdot \mathrm{g}^{-1}$ & - & - & - & [77] \\
\hline $\mathrm{RuO}_{2} \cdot \mathrm{xH}_{2} \mathrm{O} / \mathrm{CB}$ & $\begin{array}{l}\text { Novel incipient } \\
\text { wetness }\end{array}$ & $647 \mathrm{~F} \cdot \mathrm{g}^{-1}$ & - & - & - & [78] \\
\hline $\begin{array}{c}\mathrm{Co}, \mathrm{Mn}, \mathrm{Cu}, \mathrm{Fe}, \\
\mathrm{Zn} \text {-doped carbon } \\
\text { aerogels }\end{array}$ & Impregnation & $\begin{array}{l}100 \mathrm{~F} \cdot \mathrm{g}^{-1}(\mathrm{Co}) \\
107 \mathrm{~F} \cdot \mathrm{g}^{-1}(\mathrm{Mn})\end{array}$ & - & - & - & [79] \\
\hline $\mathrm{MoO}_{3} / \mathrm{AC}$ & Impregnation & $177 \mathrm{~F} \cdot \mathrm{g}^{-1}$ & - & - & $\begin{array}{c}94 \% \\
\text { (after 20,000 } \\
\text { cycles) }\end{array}$ & [80] \\
\hline ITO/AC & $\begin{array}{c}\text { Reverse } \\
\text { precipitation }\end{array}$ & - & - & - & - & [81] \\
\hline $\begin{array}{c}\mathrm{WO}_{3} / \mathrm{CA} \text { carbon } \\
\text { aerogel }\end{array}$ & $\begin{array}{l}\text { Immersion- } \\
\text { calcination }\end{array}$ & $700 \mathrm{~F} \cdot \mathrm{g}^{-1}$ & - & - & $\begin{array}{c}95 \% \\
\text { (after } 4000 \\
\text { cycles) }\end{array}$ & [82] \\
\hline Graphene- $\mathrm{MnO}_{2}$ & $\begin{array}{l}\text { Self-limiting } \\
\text { deposition }\end{array}$ & $310 \mathrm{~F} \cdot \mathrm{g}^{-1}$ & - & - & - & [83] \\
\hline Mesh-like $\mathrm{Fe}_{2} \mathrm{O}_{3} / \mathrm{C}$ & $\begin{array}{l}\text { Template free } \\
\text { greener }\end{array}$ & $315 \mathrm{~F} \cdot \mathrm{g}^{-1}$ & - & $37 \mathrm{Wh} \cdot \mathrm{kg}^{-1}$ & $\begin{array}{c}88.9 \% \\
\text { (after } 1500 \\
\text { cycles) }\end{array}$ & [84] \\
\hline $\mathrm{CuO}-\mathrm{NC}$ & $\begin{array}{l}\text { Hard } \\
\text { templating }\end{array}$ & $300 \mathrm{~F} \cdot \mathrm{g}^{-1}$ & - & - & $\begin{array}{c}91 \% \\
\text { (after } 1000 \\
\text { cycles) }\end{array}$ & [85] \\
\hline $\mathrm{Co}(\mathrm{OH})_{2 /} \mathrm{USY}$ & $\begin{array}{l}\text { Chemical } \\
\text { precipitation }\end{array}$ & $958 \mathrm{~F} \cdot \mathrm{g}^{-1}$ & - & - & J & [86] \\
\hline $\mathrm{NiO} / \mathrm{CNT}$ & Hydrothermal & $1329 \mathrm{~F} \cdot \mathrm{g}^{-1}$ & - & - & - & [87] \\
\hline $\begin{array}{c}\text { Graphene } / \mathrm{Co}_{3} \mathrm{O}_{4} \\
\text { nanowire }\end{array}$ & Hydrothermal & $1100 \mathrm{~F} \cdot \mathrm{g}^{-1}$ & - & - & - & [88] \\
\hline $\begin{array}{c}\text { Graphene } \\
\text { sheets } / \mathrm{Ag}_{2} \mathrm{~S}\end{array}$ & Solvothermal & $1063 \mathrm{~F} \cdot \mathrm{g}^{-1}$ & - & - & - & [89] \\
\hline $\mathrm{Ni}_{3} \mathrm{~S}_{2} / \mathrm{MWCNT}$ & Hydrothermal & $800 \mathrm{~F} \cdot \mathrm{g}^{-1}$ & $798 \mathrm{~W} \cdot \mathrm{kg}^{-1}$ & $19.8 \mathrm{Wh} \cdot \mathrm{kg}^{-1}$ & $\begin{array}{c}90 \% \\
\text { (after } 5000 \\
\text { cycles) }\end{array}$ & [90] \\
\hline$\alpha-\mathrm{Ni}(\mathrm{OH})_{2}-\mathrm{GO}$ & Hydrothermal & $1760.7 \mathrm{~F} \cdot \mathrm{g}^{-1}$ & - & - & - & [91] \\
\hline $\mathrm{BiMn}_{2} \mathrm{O}_{5}-\mathrm{MWCNT}$ & Hydrothermal & $540 \mathrm{~F} \cdot \mathrm{g}^{-1}$ & $3.6 \mathrm{~kW} \mathrm{~kg}^{-1}$ & $13 \mathrm{Wh} \mathrm{kg}^{-1}$ & - & [92] \\
\hline Co-Al LDH-NS/GO & LBL & $880 \mathrm{~F} \cdot \mathrm{g}^{-1}$ & - & - & - & [93] \\
\hline $\mathrm{CoMoO}_{4} /$ graphene & Hydrothermal & $394.5 \mathrm{~F} \cdot \mathrm{g}^{-1}$ & $197.2 \mathrm{~W} \cdot \mathrm{kg}^{-1}$ & $54.8 \mathrm{Wh} \cdot \mathrm{kg}^{-1}$ & - & [94] \\
\hline
\end{tabular}


Table 4. Cont.

\begin{tabular}{|c|c|c|c|c|c|c|}
\hline Materials & Method & $\begin{array}{c}\text { Specific } \\
\text { Capacitance } \\
\text { Density }\end{array}$ & $\begin{array}{l}\text { Specific Power } \\
\text { Density }\end{array}$ & $\begin{array}{c}\text { Specific Energy } \\
\text { Density }\end{array}$ & $\begin{array}{c}\text { Specific } \\
\text { Capacitance } \\
\text { Retention }\end{array}$ & Ref. \\
\hline $\mathrm{NG}-\mathrm{NiMnO}_{3}$ & Hydrothermal & $750.2 \mathrm{~F} \cdot \mathrm{g}^{-1}$ & & & - & [95] \\
\hline $\mathrm{Co}_{3} \mathrm{~S}_{4}-\mathrm{NG}$ & Hydrothermal & $2427 \mathrm{~F} \cdot \mathrm{g}^{-1}$ & & & - & [96] \\
\hline $\begin{array}{c}\mathrm{NiV}_{2} \mathrm{O}_{6} / \mathrm{Ni}\left(\mathrm{HCO}_{3}\right)_{2} \\
\text { nanoflake arrays }\end{array}$ & Hydrothermal & $7.94 \mathrm{~F} \cdot \mathrm{cm}^{-2}$ & $4.983 \mathrm{~mW} \cdot \mathrm{cm}^{-2}$ & $\begin{array}{c}0.415 \\
\mathrm{mWh} \cdot \mathrm{cm}^{-2}\end{array}$ & $\begin{array}{c}106.2 \% \\
\text { (after 10,000 } \\
\text { cycles) }\end{array}$ & [97] \\
\hline $\begin{array}{l}\mathrm{Mn} / \mathrm{PbO}_{\mathrm{x}} \\
\mathrm{Mn} / \mathrm{NiO}_{\mathrm{x}}\end{array}$ & $\begin{array}{l}\text { Chemical } \\
\text { reduction }\end{array}$ & $\begin{array}{l}185 \mathrm{~F} \cdot \mathrm{g}^{-1} \\
210 \mathrm{~F} \cdot \mathrm{g}^{-1}\end{array}$ & & & 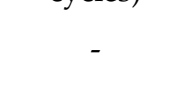 & [98] \\
\hline $\mathrm{Co}_{3} \mathrm{O}_{4} / \mathrm{Ni}(\mathrm{OH})_{2} \mathrm{O}$ & $\begin{array}{l}\text { Electrochemical } \\
\text { deposition }\end{array}$ & $1144 \mathrm{~F} \cdot \mathrm{g}^{-1}$ & & & - & [99] \\
\hline $\begin{array}{c}\mathrm{NiMoO}_{4} / \mathrm{CoMoO}_{4} \\
\text { nanorods }\end{array}$ & Hydrothermal & $1164 \mathrm{~F} \cdot \mathrm{g}^{-1}$ & $3750 \mathrm{~W} \cdot \mathrm{kg}^{-1}$ & $17.5 \mathrm{Wh} \cdot \mathrm{kg}^{-1}$ & $\begin{array}{c}87.5 \% \\
\text { (after } 3000 \\
\text { cycles) }\end{array}$ & [100] \\
\hline Ag QDs $/ \mathrm{NiMoO}_{4}$ & $\begin{array}{l}\text { Dipping and } \\
\text { drying }\end{array}$ & $3342.7 \mathrm{~F} \cdot \mathrm{g}^{-1}$ & $212.5 \mathrm{~kW} \cdot \mathrm{kg}^{-1}$ & $48.5 \mathrm{Wh} \cdot \mathrm{kg}^{-1}$ & r & [101] \\
\hline $\begin{array}{c}\text { Ppy@NiCo } \mathrm{S}_{4} \\
\text { core-shell } \\
\text { heterostructure }\end{array}$ & Hydrothermal & $908.1 \mathrm{~F} \cdot \mathrm{g}^{-1}$ & $160 \mathrm{~W} \cdot \mathrm{kg}^{-1}$ & $50.82 \mathrm{Wh} \cdot \mathrm{kg}^{-1}$ & $\begin{array}{c}126.6 \% \\
\text { (after } 2000 \\
\text { cycle) }\end{array}$ & [102] \\
\hline $\begin{array}{c}\mathrm{Co} @ \mathrm{Co}_{3} \mathrm{O}_{4} \text { core-shell } \\
\text { 3DN }\end{array}$ & $\begin{array}{l}\text { Surface } \\
\text { oxidating }\end{array}$ & $1049 \mathrm{~F} \cdot \mathrm{g}^{-1}$ & - & - & - & [103] \\
\hline $\begin{array}{c}\text { Core-shell hollow } \\
\text { CoMoS }{ }_{4} @ \text { Ni-Co-S } \\
\text { nanotubes }\end{array}$ & $\begin{array}{l}\text { Hydrothermal } \\
\text { Electrodeposition }\end{array}$ & $2208.5 \mathrm{~F} \cdot \mathrm{g}^{-1}$ & $800 \mathrm{~W} \cdot \mathrm{kg}^{-1}$ & $49.1 \mathrm{Wh} \cdot \mathrm{kg}^{-1}$ & $\begin{array}{c}90.3 \% \\
\text { (after 10,000 } \\
\text { cycles) }\end{array}$ & [104] \\
\hline
\end{tabular}

\section{Materials Based on CC}

A summary of the electrochemical performance of electrode materials based on CC is provided in Table 5. Rowlands et al. [105] concluded that CC as an electrode exhibits a specific capacitance of $35 \mathrm{~F} \cdot \mathrm{g}^{-1}$. It is worth mentioning that Dai et al. [106] developed hierarchical porous hollow $\mathrm{N}$-doped CC as an electrode material for organic-electrolyte supercapacitors, overcoming the shortcomings of pure CC with superior stability $(98 \%$ capacitance retention over 20,000 cycles). Compared with electrode materials without a substrate, the advantages of materials based on CC are reflected in the following points. The compound can grow in situ on CC and extend outward, and the growth is more uniform, resulting in a larger surface area and porous structure, shortening the ion-diffusion channel, accelerating the transmission path of electrons and ions, accelerating the Faraday capacitance reaction, and enhancing the electrolysis of liquid and electrode materials. CC also exhibits good flexibility and plasticity, as observed in the digital photograph of commercial CC, with an area of $1 \times 4 \mathrm{~cm}^{2}$ (Figure 2a). As shown in Figure $2 \mathrm{~b}$, Liu et al. [107] synthesized uniform honeycomb-like $\mathrm{NiCo}_{2} \mathrm{~S}_{4}$ nanosheets grown on CC with an excellent capacitance up to $1638 \mathrm{~F} \cdot \mathrm{g}^{-1}$ at $1 \mathrm{~A} \cdot \mathrm{g}^{-1}$. Graphene quantum dots on carbon cloth can somewhat improve specific capacitance over carbon cloth and maintain perfect flexibility, but its performance is far from adequate [10]. Zhao et al. [108] prepared a metal-organic framework using an etching/ion-exchange method, as shown in Figure 2c; the obtained ultra-thin nanosheet arrays with a mesoporous structure exhibited good conductivity with abundant active sites, resulting in enhanced electrochemical performance and a high specific capacitance of $2392 \mathrm{~F} \cdot \mathrm{g}^{-1}$ at a current density of $1 \mathrm{~A} \cdot \mathrm{g}^{-1}$. A Ni-Co$\mathrm{S} / \mathrm{ACC} / / \mathrm{AC}$ asymmetrical flexible supercapacitor exhibited good cycling stability $(82 \%$ retention after 10,000 cycles). The tortoise-shell-like structure $\alpha-\mathrm{Fe}_{2} \mathrm{O}_{3} / \mathrm{C}$ nanoarray on $\mathrm{CC}$ is shown in Figure 2d. The hollow porous structure of the $\alpha-\mathrm{Fe}_{2} \mathrm{O}_{3} / \mathrm{C}$ nanoarray, carbon nanoparticles, and numerous mesopores between the $\alpha-\mathrm{Fe}_{2} \mathrm{O}_{3}$ nanocrystals and carbon nanoparticles all improved the electrochemical performance [109]. The hierarchical $\mathrm{NiCo}_{2} \mathrm{~S}_{4} @ \mathrm{NiCo}_{x} \mathrm{~S}_{\mathrm{y}}$ core/shell nanoarrays grown on CC shown in Figure 2e and hierarchical tectorum-like $\alpha-\mathrm{Fe}_{2} \mathrm{O}_{3} /$ PPy nanoarrays grown on CC shown in Figure $2 \mathrm{f}$ exhibited areal 
capacitances as high as $3.9 \mathrm{~F} \cdot \mathrm{cm}^{-2}$ at $1 \mathrm{~mA} \cdot \mathrm{cm}^{-2}$ and $382.4 \mathrm{mF} \cdot \mathrm{cm}^{-2}$ at $0.5 \mathrm{~mA} \cdot \mathrm{cm}^{-2}$, respectively $[110,111]$. Besides above advantages, electrode materials based on CC have more active sites, increasing the energy storage and capacitance [112-114].

The use of base materials such as CC for constructing nano-sized forms is an effective way to improve the electrochemical performance of electrode materials by shortening the length of the ion-diffusion channel [115-120]. Horng et al. [121] prepared nano-sized polyaniline nanowires on CC (PANI-NWs/CC) via an electrochemical method; the materials exhibited specific capacitance up to $1220 \mathrm{~F} \cdot \mathrm{g}^{-1}$ while overcoming the cycle degradation issues caused by mechanical issues. The one-dimensional structure of the metal nanowires as the supporting framework of the fixing material effectively produced a porous structure and improved the performance and stability of the materials with good conductivity and fast charge transport [122,123].

The preparation methods of electrode materials consist of electrochemical deposition, chemical deposition, dip dyeing, and hydrothermal methods. Compounds obtained under hydrothermal conditions can overcome the hard agglomeration of certain high-temperature preparation methods. The characteristics of hydrothermally produced materials include high particle purity, small size (nano-level), good dispersion, uniform distribution, no agglomeration, good controllability, low production cost, and environmentally friendly reaction conditions and atmosphere. In addition, from the perspective of environmental protection, the hydrothermal method is a mild, green, low-cost, and easy-to-operate preparation method. It has become an important choice for the preparation of electrode materials after ongoing improvement.

Figure 3 presents SEM images of multiple metal sulfides based on CC prepared via the hydrothermal method; a sea-urchin-like $\mathrm{Ni}-\mathrm{Co}-\mathrm{S}$ compound on $\mathrm{CC}$ with a high surface area and many active sites is displayed in Figure 3a. However, it can be observed that the high surface energy results in local agglomeration. After improving the experimental conditions, a good structure of nano-needle nickel cobalt sulfide growing on CC with excellent electrochemical performance was obtained (Figure 3b). In Figure 3c, a small amount of Fe element was added under the same experimental atmosphere as Figure 3b; the $\mathrm{Ni}-\mathrm{Co}-\mathrm{Fe}-\mathrm{S}$ nanoparticles are uniformly distributed on the carbon cloth with scarce agglomeration of large particles.

a
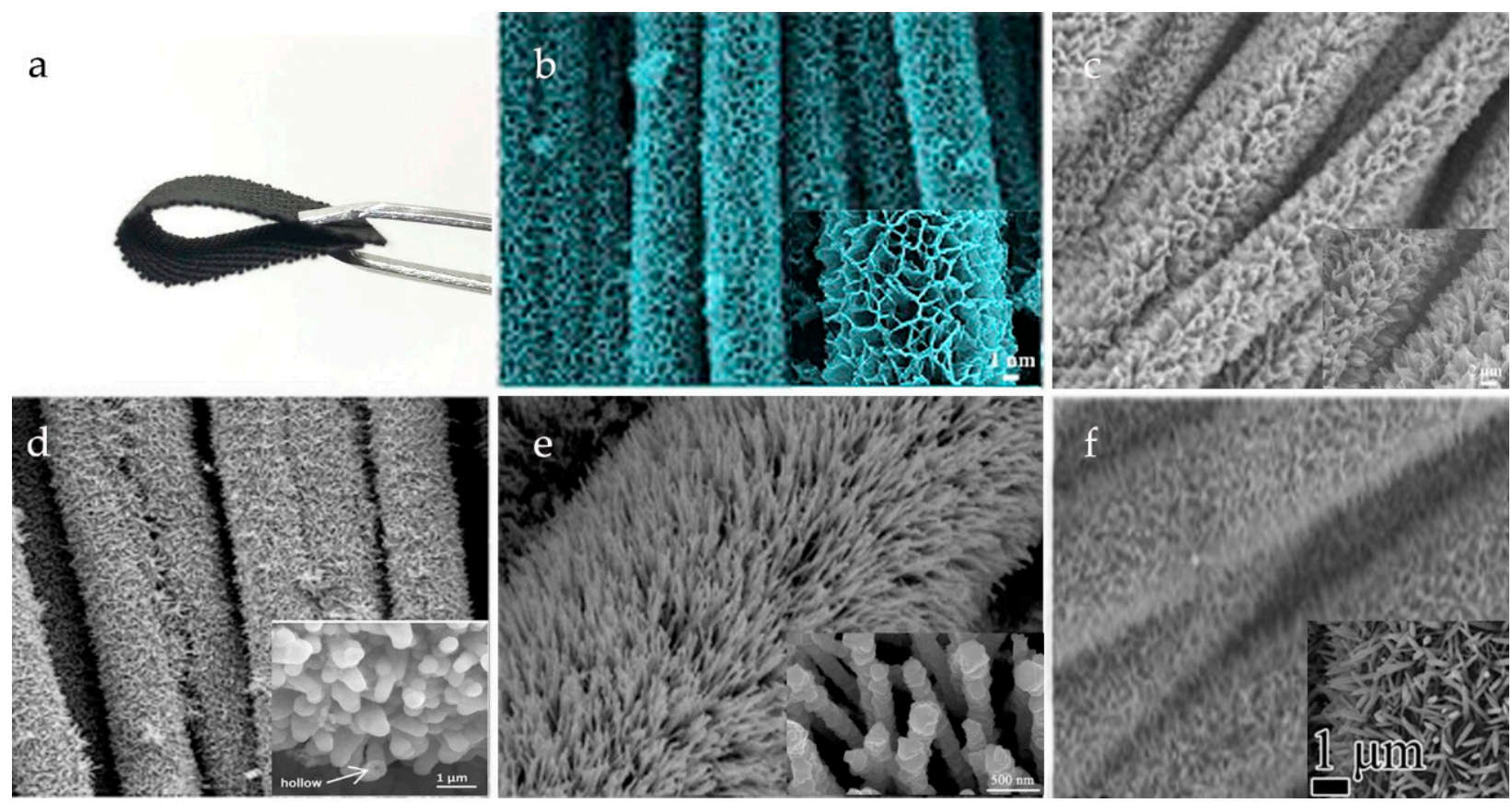

Figure 2. (a) Digital photograph of commercial CC with area of $1 \times 4 \mathrm{~cm}^{2}$; (b-f) SEM images of several compounds with different structures grown on CC [107-111]. Adapted from [108-111], with permission from Elsevier, 2020. 


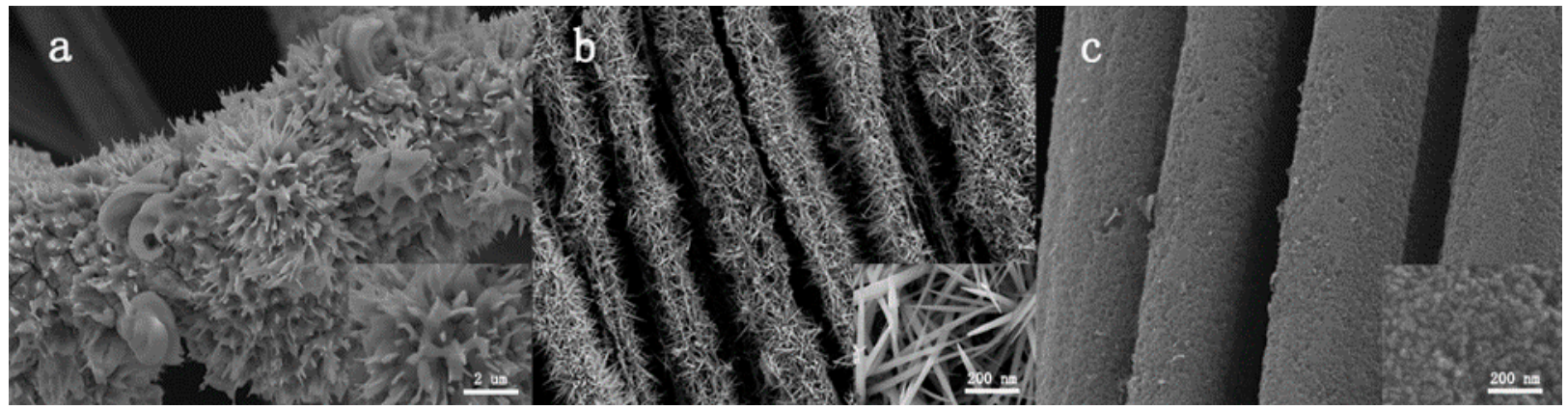

Figure 3. SEM images of (a) sea-urchin-like Ni-Co-S compound on CC, (b) Ni-Co-S nano-needle on CC, and (c) Ni-Co-Fe-S nanoparticles on CC.

Table 5. Properties of materials based on CC for supercapacitors prepared by various methods.

\begin{tabular}{|c|c|c|c|c|c|c|}
\hline Materials & Method & $\begin{array}{c}\text { Specific } \\
\text { Capacitance } \\
\text { Density }\end{array}$ & $\begin{array}{l}\text { Specific Power } \\
\text { Density }\end{array}$ & $\begin{array}{c}\text { Specific Energy } \\
\text { Density }\end{array}$ & $\begin{array}{c}\text { Specific } \\
\text { Capacitance } \\
\text { Retention }\end{array}$ & Ref. \\
\hline $\mathrm{CC}$ & $\begin{array}{l}\text { Electrochemical } \\
\text { anodization }\end{array}$ & $35 \mathrm{~F} \cdot \mathrm{g}^{-1}$ & - & - & - & [105] \\
\hline $\begin{array}{c}\text { N-doped activated } \\
\text { CC }\end{array}$ & $\begin{array}{c}\text { One-step } \\
\text { etching \& } \\
\text { doping }(\mathrm{E} \& D)\end{array}$ & $215.9 \mathrm{~F} \cdot \mathrm{g}^{-1}$ & - & - & $\begin{array}{c}98 \% \\
(20,000 \text { cycles })\end{array}$ & [106] \\
\hline $\begin{array}{c}\text { Graphene } \\
\text { Quantum Dots } \\
\text { (GQD)/CC }\end{array}$ & $\begin{array}{c}\text { Peroxide- } \\
\text { assisted } \\
\text { hydrothermal }\end{array}$ & $70 \mathrm{mF} \cdot \mathrm{cm}^{-2}$ & & & & [10] \\
\hline $\mathrm{NiCO}_{2} \mathrm{~S}_{4} / \mathrm{CC}$ & Hydrothermal & $1638 \mathrm{~F} \cdot \mathrm{g}^{-1}$ & $799.6 \mathrm{~W} \cdot \mathrm{kg}^{-1}$ & $25.2 \mathrm{Wh} \cdot \mathrm{kg}^{-1}$ & - & [107] \\
\hline $\mathrm{Ni}-\mathrm{Co}-\mathrm{S} / \mathrm{ACC}$ & Hydrothermal & $2392 \mathrm{~F} \cdot \mathrm{g}^{-1}$ & $800.2 \mathrm{~W} \cdot \mathrm{kg}^{-1}$ & $30.1 \mathrm{Wh} \cdot \mathrm{kg}^{-1}$ & $\begin{array}{c}82 \%(10,000 \\
\text { cycles })\end{array}$ & [108] \\
\hline $\begin{array}{c}\alpha-\mathrm{Fe}_{2} \mathrm{O}_{3} / \mathrm{C} \\
\text { nanoarrays on } \mathrm{CC}\end{array}$ & Hydrothermal & $391.8 \mathrm{~F} \cdot \mathrm{g}^{-1}$ & - & - & $\begin{array}{l}91.8 \%(4000 \\
\text { cycles })\end{array}$ & [109] \\
\hline $\begin{array}{c}\mathrm{NiCo}_{2} \mathrm{~S}_{4} @ \mathrm{NiCoxSy} \\
\text { on } \mathrm{CC}\end{array}$ & Hydrothermal & $3.9 \mathrm{~F} \cdot \mathrm{cm}^{-2}$ & - & - & - & [110] \\
\hline $\begin{array}{c}\alpha-\mathrm{Fe}_{2} \mathrm{O}_{3} / \mathrm{PPy} \\
\text { nanoarrays on } \mathrm{CC}\end{array}$ & $\begin{array}{l}\text { Hydrothermal } \\
\text { and in situ } \\
\text { vapor-phase } \\
\text { polymerization }\end{array}$ & $382.4 \mathrm{mF} \cdot \mathrm{cm}^{-2}$ & - & - & - & [111] \\
\hline $\begin{array}{c}\text { Hierarchical } \\
\mathrm{Co}(\mathrm{OH})_{2} @ \\
\mathrm{NiMoS}_{4} \text { on } \mathrm{CC}\end{array}$ & Hydrothermal & $2229 \mathrm{~F} \cdot \mathrm{g}^{-1}$ & $1000 \mathrm{~W} \cdot \mathrm{kg}^{-1}$ & $159.5 \mathrm{Wh} \cdot \mathrm{kg}^{-1}$ & $\begin{array}{c}100 \%(5000 \\
\text { cycles) }\end{array}$ & [112] \\
\hline $\mathrm{ZIF}-67$ on $\mathrm{CC}$ & Dipping & $829 \mathrm{~F} \cdot \mathrm{g}^{-1}$ & - & - & $\begin{array}{c}103 \%(15,000 \\
\text { cycles })\end{array}$ & [113] \\
\hline ROCC@PDAA on CC & $\begin{array}{l}\text { In-situ chemical } \\
\text { oxidation } \\
\text { polymerization }\end{array}$ & $81.9 \mathrm{~F} \cdot \mathrm{g}^{-1}$ & - & - & $\begin{array}{c}159 \%(20,000 \\
\text { cycles })\end{array}$ & [114] \\
\hline $\begin{array}{l}\mathrm{NiO} \text { nanoflake arrays } \\
\text { on } \mathrm{CC}\end{array}$ & CBD & $660 \mathrm{~F} \cdot \mathrm{g}^{-1}$ & - & - & $\begin{array}{l}82 \% \text { ( } 4000 \\
\text { cycles) }\end{array}$ & [115] \\
\hline $\mathrm{MoO}_{3}$ film on $\mathrm{CC}$ & Electrodeposition & $835 \mathrm{~F} \cdot \mathrm{g}^{-1}$ & $1000 \mathrm{~W} \cdot \mathrm{kg}^{-1}$ & $78 \mathrm{Wh} \cdot \mathrm{kg}^{-1}$ & $\begin{array}{c}98 \%(8000 \\
\text { cycles) }\end{array}$ & [116] \\
\hline $\begin{array}{c}\mathrm{MoS}_{2} \text { nanospheres } \\
\text { on CC }\end{array}$ & Hydrothermal & $368 \mathrm{~F} \cdot \mathrm{g}^{-1}$ & $128 \mathrm{~W} \cdot \mathrm{kg}^{-1}$ & $5.42 \mathrm{Wh} \cdot \mathrm{kg}^{-1}$ & $\begin{array}{l}96.5 \% \text { (after } \\
5000 \text { cycles) }\end{array}$ & [117] \\
\hline
\end{tabular}


Table 5. Cont.

\begin{tabular}{|c|c|c|c|c|c|c|}
\hline Materials & Method & $\begin{array}{c}\text { Specific } \\
\text { Capacitance } \\
\text { Density }\end{array}$ & $\begin{array}{l}\text { Specific Power } \\
\text { Density }\end{array}$ & $\begin{array}{c}\text { Specific Energy } \\
\text { Density }\end{array}$ & $\begin{array}{c}\text { Specific } \\
\text { Capacitance } \\
\text { Retention }\end{array}$ & Ref. \\
\hline $\begin{array}{c}\mathrm{Ni}(\mathrm{OH})_{2} \text { on } \mathrm{CC} \\
\mathrm{Co}(\mathrm{OH}) \times \mathrm{CO}_{3} \text { on } \mathrm{CC}\end{array}$ & Hydrothermal & $\begin{array}{l}789 \mathrm{~F} \cdot \mathrm{g}^{-1} \\
550 \mathrm{~F} \cdot \mathrm{g}^{-1}\end{array}$ & $1.4 \mathrm{~kW} \cdot \mathrm{kg}^{-1}$ & $\begin{array}{c}41.1 \mathrm{Wh} \cdot \mathrm{kg}^{-1} \\
33.48 \mathrm{Wh} \cdot \mathrm{kg}^{-1}\end{array}$ & $\begin{array}{c}98 \% ; 97.6 \% \\
\text { (after } 5000 \\
\text { cycles) }\end{array}$ & [118] \\
\hline $\begin{array}{c}\text { P-doped } \mathrm{NiCo}_{2} \mathrm{~S}_{4} \\
\text { nanotube arrays on } \\
\text { CC }\end{array}$ & Hydrothermal & $8.03 \mathrm{~F} \cdot \mathrm{cm}^{-2}$ & $750 \mathrm{~W} \cdot \mathrm{kg}^{-1}$ & $42.1 \mathrm{Wh} \cdot \mathrm{kg}^{-1}$ & $\begin{array}{c}87.5 \%(5000 \\
\text { cycles) }\end{array}$ & [119] \\
\hline $\begin{array}{c}\text { NCLP@ } \\
\text { NiMn-LDH on CC }\end{array}$ & Hydrothermal & $2318 \mathrm{~F} \cdot \mathrm{g}^{-1}$ & $750 \mathrm{~W} \cdot \mathrm{kg}^{-1}$ & $42.2 \mathrm{~W} \mathrm{~h} \mathrm{~kg}^{-1}$ & - & [120] \\
\hline PANI-NWs/CC & $\begin{array}{l}\text { Electrochemical } \\
\text { polymerization }\end{array}$ & $1079 \mathrm{~F} \cdot \mathrm{g}^{-1}$ & $12.1 \mathrm{~kW} \cdot \mathrm{kg}^{-1}$ & $100.9 \mathrm{Wh} \cdot \mathrm{kg}^{-1}$ & - & [121] \\
\hline $\begin{array}{c}\text { NC LDH } \\
\text { NSs@Ag@CC } \\
\text { Ni-Co LDH }\end{array}$ & $\begin{array}{l}\text { Electrochemical } \\
\text { deposition }\end{array}$ & $\begin{array}{c}1133.3 \\
\mathrm{mF} \cdot \mathrm{cm}^{-2}\end{array}$ & - & - & $\begin{array}{l}80.47 \%(2000 \\
\text { cycles })\end{array}$ & [122] \\
\hline $\begin{array}{c}\text { Nanoflakes- } \mathrm{ZnO} \\
\text { nanowires hybrid } \\
\text { array on } \mathrm{CC}\end{array}$ & Hydrothermal & $927 \mathrm{~F} \cdot \mathrm{g}^{-1}$ & $46.15 \mathrm{~kW} \cdot \mathrm{kg}^{-1}$ & $45.55 \mathrm{Wh} \cdot \mathrm{kg}^{-1}$ & $\begin{array}{c}96.02 \%(3000 \\
\text { cycles })\end{array}$ & [123] \\
\hline
\end{tabular}

\section{Main Existing Issues, Corresponding Solutions, and Future Work}

Although electrode materials exhibit great potential due to low cost, high performance, and good flexibility, many unanswered questions remain. Among the electrode materials, carbon materials with multidimensional pore characteristics, graphene, and atom-doped carbon materials have developed dramatically. In the process of basic research on electrode materials, various advanced design concepts have driven the rapid development of related fields and industries.

However, low load is completely inadequate to meet the needs of commercial electrodes. Therefore, increasing the active material load of the electrode and further exploring and optimizing its electrochemical performance will provide a reference and guide for its future practical application.

Advanced technology was used to reveal the phase evolution law of materials and the structure-effect relationship between structure and properties, so as to guide the design and construction of electrode materials. We can see the future trend of electrode materials from bionic carbon materials, but the huge technical challenges are still difficult problems.

As a substrate growth material to construct a variety of three-dimensional structural composite materials, especially with the redox properties of transition metals to build porous structures with high specific surface area, the performance of composite materialbased carbon materials are surprising.

For three-dimensional composite materials with a controllable nanostructure based on CC, the most important thing is that they provide more active sites and exhibit excellent electrochemical properties [124-127]. We can see that metal composites exhibit oxidative performance, which is the advantage that other materials cannot achieve. However, the controllability and stability of the structure are large problems. When the growth dimension reaches nano-level, especially, the structure has a larger surface area, there are more active sites, and at the same time the surface energy is greater, agglomeration is easier, and the structure can be more easily damaged. The hydrothermal method not only does not destroy the structure and performance of the $\mathrm{CC}$, but also provides a mild environment for the growth of composite material based on CC, and three-dimensional nano-structures exhibit more possibilities and potential. 


\section{Conclusions}

Supercapacitors have the advantages of high power density, fast charge and discharge rates, long cycle life, high safety, and reliability as a new generation of green energy materials to replace traditional energy materials. The hydrothermal method has the characteristics of simplicity, environmental friendliness, and uniform growth of the load. Nanostructures can be grown directly on CC via a hydrothermal method, including nano-needles, tree-like and nano-flower shapes, ultra-thin nanosheets, and tectorum-like shapes, which have high surface areas and porous structures, shorten the transmission channels of ions and electrons, and increase the active sites for the Faraday capacitance reactions. The synergistic effect between the components also effectively improves their specific capacitance, cycle stability, and rate capability. In addition, flexible supercapacitors assembled with electrode materials based on CC have great advantages for the energy supply for wearable devices.

Author Contributions: Figures and tables, X.W.; investigation, X.W. and P.X.; resources, X.W., P.Z. and S.M.; writing—original draft preparation, X.W., P.Z. and S.M.; writing-review and editing, P.X. and X.W.; supervision, P.X.; project administration, X.W. All authors have read and agreed to the published version of the manuscript.

Funding: This research received no external funding.

Institutional Review Board Statement: Not applicable.

Informed Consent Statement: Not applicable.

Data Availability Statement: Not applicable.

Conflicts of Interest: The authors declare no conflict of interest.

\section{References}

1. Liu, C.; Li, F.; Ma, L.; Cheng, H. Advanced Materials for Energy Storage. Adv. Mater. 2010, 22, E28-E62. [CrossRef]

2. Wang, X.; Li, M.; Chang, Z.; Yang, Y.; Wu, Y.; Liu, X. $\mathrm{Co}_{3} \mathrm{O}_{4} @ M W C N T$ Nanocable as Cathode with Superior Electrochemical Performance for Supercapacitors. ACS Appl. Mater. Interfaces 2015, 7, 2280-2285. [CrossRef] [PubMed]

3. Aricò, A.S.; Bruce, P.; Scrosati, B.; Tarascon, J.M.; van Schalkwijk, W. Nanostructured materials for advanced energy conversion and storage devices. Nat. Mater. 2005, 4, 366-377. [CrossRef] [PubMed]

4. González, A.; Goikolea, E.; Barrena, J.A.; Mysyk, R. Review on supercapacitors: Technologies and materials. Renew. Sustain. Energy Rev. 2016, 58, 1189-1206. [CrossRef]

5. Choi, J.W.; Aurbach, D. Promise and reality of post-lithium-ion batteries with high energy densities. Nat. Rev. Mater. 2016, 1, 359-367. [CrossRef]

6. Miller, J.R.; Simon, P. Electrochemical capacitors for energy management. Science 2008, 321, 651-652. [CrossRef]

7. Nassif, G.G.; de Almeida, S.C.A. Impact of powertrain hybridization on the performance and costs of a fuel cell electric vehicle. Int. J. Hydrogen Energy 2020, 45, 21722-21737. [CrossRef]

8. Hu, W.; Xiang, R.; Lin, J.; Cheng, Y.; Lu, C. Lignocellulosic Biomass-Derived Carbon Electrodes for Flexible Supercapacitors: An Overview. Materials 2021, 14, 4571. [CrossRef]

9. dos Reis, G.S.; de Oliveira, H.P.; Larsson, S.H.; Thyrel, M.; Claudio Lima, E. A Short Review on the Electrochemical Performance of Hierarchical and Nitrogen-Doped Activated Biocarbon-Based Electrodes for Supercapacitors. Nanomaterials 2021, 11, 424. [CrossRef]

10. Tjandra, R.; Liu, W.; Zhang, M.; Yu, A. All-carbon flexible supercapacitors based on electrophoretic deposition of graphene quantum dots on carbon cloth. J. Power Sources 2019, 438, 227009. [CrossRef]

11. Li, Y.; Zijll, M.; Chiang, S.; Pan, N. KOH modified graphene nanosheets for supercapacitor electrodes. J. Power Sources 2011, 196, 6003-6006. [CrossRef]

12. Zhai, Y.; Dou, Y.; Zhao, D.; Fulvio, P.F.; Mayes, R.T.; Dai, S. Carbon Materials for Chemical Capacitive Energy Storage. Adv. Mater. 2011, 23, 4828-4850. [CrossRef] [PubMed]

13. Sevilla, M.; Mokaya, R. Energy storage applications of activated carbons: Supercapacitors and hydrogen storage. Energy Environ. Sci. 2014, 7, 1250-1280. [CrossRef]

14. Jin, H.; Feng, X.; Li, J.; Li, M.; Xia, Y.; Yuan, Y.; Yang, C.; Dai, B.; Lin, Z.; Wang, J.; et al. Heteroatom-doped porous carbon materials with unprecedented high volumetric capacitive performance. Angew. Chem. Int. Ed. 2019, 58, 2397-2401. [CrossRef] [PubMed]

15. Song, Z.; Li, L.; Zhu, D.; Miao, L.; Duan, H.; Wang, Z.; Xiong, W.; Lv, Y.; Liu, M.; Gan, L. Synergistic design of a N, O co-doped honeycomb carbon electrode and an ionogel electrolyte enabling all-solid-state supercapacitors with an ultrahigh energy density. J. Mater. Chem. 2019, 7, 816-826. [CrossRef] 
16. Jiang, J.; Li, Y.; Liu, J.; Huang, X.; Yuan, C.; Wen, X. Recent Advances in Metal Oxide-based Electrode Architecture Design for Electrochemical Energy Storage. Adv. Mater. 2012, 24, 5166-5180. [CrossRef] [PubMed]

17. Ding, K.; Zhang, X.; Yang, P.; Cheng, X. A precursor-derived morphology-controlled synthesis method for mesoporous $\mathrm{Co}_{3} \mathrm{O}_{4}$ nanostructures towards supercapacitor application. CrystEngComm 2016, 18, 8253-8261. [CrossRef]

18. Shanmugavani, A.; Selvan, R.K. Improved electrochemical performances of $\mathrm{CuCo}_{2} \mathrm{O}_{4} / \mathrm{CuO}$ nanocomposites for asymmetric supercapacitors. Electrochim. Acta 2016, 188, 852-862. [CrossRef]

19. Marsan, B.; Fradette, N.; Beaudoin, G. Physicochemical and Electrochemical Properties of $\mathrm{CuCo}_{2} \mathrm{O}_{4}$ Electrodes Prepared by Thermal Decomposition for Oxygen Evolution. J. Electrochem. Soc. 1992, 139, 1889-1896. [CrossRef]

20. Snook, G.A.; Kao, P.; Best, A.S. Conducting-polymer-based supercapacitor devices and electrodes. J. Power Sources 2010, 196, 12. [CrossRef]

21. Li, W.; Lu, H.; Zhang, N.; Ma, M. Enhancing the Properties of Conductive Polymer Hydrogels by Freeze-Thaw Cycles for High-Performance Flexible Supercapacitors. ACS Appl. Mater. Interfaces 2017, 9, 20142-20149. [CrossRef]

22. Ginting, R.T.; Ovhal, M.M.; Kang, J.W. A Novel Design of Hybrid Transparent Electrodes for High Performance and Ultra-Flexible Bifunctional Electrochromic-Supercapacitors. Nano Energy 2018, 53, 650-657. [CrossRef]

23. Li, X.; Gao, Y.; Yang, G. A flexible, transparent and super-long-life supercapacitor based on ultrafine $\mathrm{Co}_{3} \mathrm{O}_{4}$ nanocrystal electrodes. Nanoscale 2016, 8, 4227-4235. [CrossRef] [PubMed]

24. Shen, L.; Du, L.; Tan, S.; Zang, Z.; Zhao, C.; Mai, W. Flexible electrochromic supercapacitor hybrid electrodes based on tungsten oxide films and silver nanowires. Chem. Commun. 2016, 52, 6296-6299. [CrossRef]

25. Sial, Q.A.; Javed, M.S.; Lee, Y.J.; Duy, L.T.; Seo, H. Flexible and transparent graphene-based supercapacitors decorated with nanohybrid of tungsten oxide nanoflakes and nitrogen-doped-graphene quantum dots. Ceram. Int. 2020, 46, 23145-23154. [CrossRef]

26. Barik, R.; Tanwar, V.; Kumar, R.; Ingole, P.P. A high energy density and high rate capability flexible supercapacitor based on electro-spun highly porous $\mathrm{SnO}_{2} @$ carbon nanofibers. J. Mater. Chem. A 2020, 8, 15110-15121. [CrossRef]

27. Gamby, J.; Taberna, P.L.; Simon, P.; Fauvarque, J.F.; Chesnear, M. Studies and characterisations of various activated carbons used for carbon/carbon supercapacitors. J. Power Sources 2001, 101, 109-116. [CrossRef]

28. Beck, F.; Dolata, M.; Grivei, E.; Probst, N. Electrochemical supercapacitors based on industrial carbon blacks in aqueous $\mathrm{H}_{2} \mathrm{SO}_{4}$. J. Power Sources 2001, 31, 845-853.

29. Fischer, U.; Saliger, R.; Bock, V.; Petricevic, R.; Fricke, J. Carbon Aerogels as Electrode Material in Supercapacitors. J. Porous Mater. 1997, 4, 281-285. [CrossRef]

30. Li, W.; Reichenauer, G.; Fricke, J. Carbon aerogels derived from cresol-resorcinol-formaldehyde for supercapacitors. Carbon 2002, 40, 2955-2959. [CrossRef]

31. Frackowiak, E.; Metenier, K.; Bertagna, V.; Beguin, F. Supercapacitor electrodes from multiwalled carbon nanotubes. Appl. Phys. Lett. 2000, 77, 2421. [CrossRef]

32. Liu, C.; Bard, A.J.; Wudl, F.; Weitz, I.; Heath, J.R. Electrochemical Characterization of Films of Single-Walled Carbon Nanotubes and Their Possible Application in supercapacitors. Electrochem. Solid-State Lett. 1999, 2, 577-578. [CrossRef]

33. Pan, H.; Poh, C.K.; Feng, Y.P.; Lin, J. Supercapacitor Electrodes from Tubes-in-Tube Carbon Nanostructures. Chem. Mater. 2007, 19, 6120-6125. [CrossRef]

34. Yang, S.Y.; Chang, K.H.; Tien, H.W.; Lee, Y.F.; Li, S.M.; Wang, Y.S.; Wang, J.Y.; Ma, C.C.M.; Hu, C.C. Design and tailoring of a hierarchical graphene-carbon nanotube architecture for supercapacitors. J. Mater. Chem. 2011, 21, 2374-2380. [CrossRef]

35. You, B.; Yang, J.; Sun, Y.; Su, Q. Easy synthesis of hollow core, bimodal mesoporous shell carbon nanospheres and their application in supercapacitor. Chem. Commun. 2011, 47, 12364-12366. [CrossRef]

36. McDonough, J.K.; Frolov, A.I.; Presser, V.; Niu, J.; Miller, C.H.; Uvieto, T.; Fedorov, M.V.; Gogotsi, Y. Influence of the structure of carbon onions on their electrochemical performance in supercapacitor electrodes. Carbon 2012, 50, 3298-3309. [CrossRef]

37. Liu, B.; Hiroshi, S.; Jiang, H.; Zhang, X.; Xu, Q. Metal-organic framework (MOF) as a template for syntheses of nanoporous carbons as electrode materials for supercapacitor. Carbon 2010, 48, 456-463. [CrossRef]

38. Zhao, X.C.; Zhang, Q.; Chen, C.M.; Zhang, B.; Reiche, S.; Wang, A.; Zhang, T.; Schlögl, R.; Su, D.S. Aromatic sulfide, sulfoxide, and sulfone mediated mesoporous carbon monolith for use in supercapacitor. Nano Energy 2012, 1, 624-630. [CrossRef]

39. Shirakawa, H.; Louis, E.J.; Macdiarmid, A.G.; Chiang, A.G.; Heeger, A.J. Synthesis of Electrically Conducting Organic Polymers: Halogen Derivatives of Polyacetylene, (CH)x. J. Chem. Soc. Chem. Commun. 1977, 16, 578-580. [CrossRef]

40. Ghosh, S.; Inganäs, O. Conducting Polymer Hydrogels as 3D Electrodes: Applications for Supercapacitors. Adv. Mater. 1999, 11, 1214-1218. [CrossRef]

41. Arbizzani, C.; Mastragostino, M.; Meneghello, L. Polymer-based redox supercapacitors: A comparative study. Electrochim. Acta 1996, 41, 21-26. [CrossRef]

42. Ferraris, J.P.; Eissa, M.M.; Brotherston, I.D.; Loveday, D.C.; Moxey, A.A. Preparation and electrochemical evaluation of poly (3-phenylthiophene) derivatives: Potential materials for electrochemical capacitors. Chem. Mater. 1998, 10, 3528-3535. [CrossRef]

43. Fusalba, F.; Mehdi, N.E.; Breau, L.; Bélanger, D. Physicochemical and Electrochemical Characterization of Polycyclopenta[2,1b;3,4-b']dithiophen-4-one as an Active Electrode for Electrochemical Supercapacitors. Chem. Mater. 1999, 11, $2743-2753$. [CrossRef] 
44. Gouérec, P.; Talbi, H.; Miousse, D.; Tran-Van, F.; Dao, L.H.; Lee, K.H. Preparation and Modification of Polyacrylonitrile Microcellular Foam Films for Use as Electrodes in Supercapacitors. J. Electrochem. Soc. 2001, 148, A94-A101. [CrossRef]

45. Park, J.H.; Ko, J.M.; Park, O.O.; Kim, D.W. Capacitance properties of graphite/polypyrrole composite electrode prepared by chemical polymerization of pyrrole on graphite fiber. J. Power Sources 2002, 105, 20-25. [CrossRef]

46. Fang, Y.; Liu, J.; Yu, D.; Wicksted, J.P.; Kalkan, K.; Topal, C.O.; Flanders, B.N.; Wu, J.; Li, J. Self-supported supercapacitor membranes: Polypyrrole-coated carbon nanotube networks enabled by pulsed electrodeposition. J. Power Sources 2010, 195, 674-679. [CrossRef]

47. Dubal, D.P.; Lee, S.H.; Kim, J.G. Porous polypyrrole clusters prepared by electropolymerization for a high performance supercapacitor. J. Mater. Chem. 2012, 22, 3044-3052. [CrossRef]

48. Jyothibasu, J.P.; Lee, R.H. Green synthesis of polypyrrole tubes using curcumin template for excellent electrochemical performance in supercapacitors. J. Mater. Chem. A 2020, 8, 3186-3202. [CrossRef]

49. Fusalba, F.; Ho, H.A.; Breau, L.; Bélanger, D. Poly(Cyano-Substituted Diheteroareneethylene) as Active Electrode Material for Electrochemical Supercapacitors. Chem. Mater. 2000, 12, 2581-2589. [CrossRef]

50. Zheng, J.P.; Cygan, P.J.; Jow, T.R. Hydrous Ruthenium Oxide as an Electrode Material for Electrochemical Capacitors. J. Electrochem. Soc. 1995, 142, 2699-2703. [CrossRef]

51. Patake, V.D.; Pawar, S.M.; Shinde, V.R.; Gujar, T.P.; Lokhande, C.D. The growth mechanism and supercapacitor study of anodically deposited amorphous ruthenium oxide films. Curr. Appl. Phys. 2009, 10, 99-103. [CrossRef]

52. Wang, C.; Hu, C. Electrochemical and textural characterization of binary Ru-Sn oxides synthesized under mild hydrothermal conditions for supercapacitors. Electrochim. Acta 2005, 50, 2573-2581. [CrossRef]

53. Sugimoto, W.; Shibutani, T.; Murakami, Y.; Takasu, Y. Charge Storage Capabilities of Rutile-Type $\mathrm{RuO}_{2}-\mathrm{VO}_{2}$ Solid Solution for Electrochemical Supercapacitors. Electrochem. Solid-State Lett. 2002, 5, A170-A172. [CrossRef]

54. Conway, B.E. Transition from "Supercapacitor" to "Battery" Behavior in Electrochemical Energy Storage. J. Electrochem. Soc. 1991, 138, 1539-1548. [CrossRef]

55. Prasad, K.R.; Koga, K.; Miura, N. Electrochemical Deposition of Nanostructured Indium Oxide: High-Performance Electrode Material for Redox Supercapacitors. Chem. Mater. 2004, 16, 1845-1847. [CrossRef]

56. Nam, K.W.; Yoon, W.S.; Kim, K.B. X-ray absorption spectroscopy studies of nickel oxide thin film electrodes for supercapacitors. Electrochim. Acta 2002, 47, 3201-3209. [CrossRef]

57. Purushothaman, K.K.; Babu, I.M.; Sethuraman, B.; Muralidharan, G. Nanosheet-assembled NiO microstructures for highperformance supercapacitors. ACS Appl. Mater. Interfaces 2013, 5, 10767-10773. [CrossRef]

58. Kandalkar, S.G.; Gunjakar, J.L.; Lokhande, C.D. Preparation of cobalt oxide thin films and its use in supercapacitor application. Appl. Surf. Sci. 2008, 254, 5540-5544. [CrossRef]

59. Dubal, D.P.; Dhawale, D.S.; Salunkhe, R.R.; Jamdade, V.S.; Lokhande, C.D. Fabrication of copper oxide multilayer nanosheets for supercapacitor application. J. Alloys Compd. 2010, 492, 26-30. [CrossRef]

60. Ye, C.; Lin, Z.M.; Hui, S.Z. Electrochemical and capacitance properties of rod-shaped $\mathrm{MnO}_{2}$ for supercapacitor. J. Electrochem. Soc. 2005, 152, A1272-A1278. [CrossRef]

61. Dubala, D.P.; Dhawale, D.S.; Salunkhe, R.R.; Pawar, S.M.; Lokhande, C.D. A novel chemical synthesis and characterization of $\mathrm{Mn}_{3} \mathrm{O}_{4}$ thin films for supercapacitor application. Appl. Surf. Sci. 2010, 256, 4411-4416. [CrossRef]

62. Bao, S.J.; Li, C.M.; Guo, C.X.; Qiao, Y. Biomolecule-assisted synthesis of cobalt sulfide nanowires for application in supercapacitors. J. Power Sources 2008, 180, 676-681. [CrossRef]

63. Qian, L.; Tian, X.; Yang, L.; Mao, J.; Yuan, H.; Xiao, D. High specific capacitance of CuS nanotubes in redox active polysulfide electrolyte. RSC Adv. 2013, 3, 1703-1708. [CrossRef]

64. Geng, X.M.; Zhang, Y.L.; Han, Y.; Li, J.; Yang, L.; Benamara, M.; Chen, L.; Zhu, H. Two-dimensional water-coupled metallic MoS 2 with nanochannels for ultrafast supercapacitors. Nano Lett. 2017, 17, 1825-1832. [CrossRef]

65. Jiang, H.; Zhao, T.; Li, C.; Ma, J. Hierarchical self-assembly of ultrathin nickel hydroxide nanoflakes for high-performance supercapacitors. J. Mater. Chem. 2011, 21, 3818-3823. [CrossRef]

66. Jiang, J.; Liu, J.; Ding, R.; Zhu, J.; Li, Y.; Hu, A.; Li, X.; Huang, X. Large-scale uniform R-Co(OH $)_{2}$ long nanowire arrays grown on graphite as pseudocapacitor electrodes. ACS Appl. Mater. Interfaces 2011, 3, 99-103. [CrossRef]

67. Nithya, V.D.; Pandi, K.; Lee, Y.S.; Kalai Selvan, R. Synthesis, characterization and electrochemical performances of nanocrystalline $\mathrm{FeVO}_{4}$ as negative and $\mathrm{LiCoPO}_{4}$ as positive electrode for asymmetric supercapacitor. Electrochim. Acta 2015, 167, 97-104. [CrossRef]

68. Lu, X.F.; Wu, D.J.; Li, R.Z.; Ye, S.H.; Tong, Y.X.; Li, G.R. Hierarchical $\mathrm{NiCo}_{2} \mathrm{O}_{4}$ nanosheets@hollow microrod arrays for highperformance asymmetric supercapacitors. J. Mater. Chem. A 2014, 2, 4706-4713. [CrossRef]

69. Hu, W.; Chen, R.Q.; Xie, W.; Zou, L.; Qin, N.; Bao, D. CoNi $\mathrm{S}_{4}$ Nanosheet Arrays Supported on Ni Foams with Ultrahigh Capacitance for Aqueous Asymmetric Supercapacitor Applications. ACS Appl. Mater. Interfaces 2014, 12, 19318-19326. [CrossRef] [PubMed]

70. Yu, Y.W.; Tan, Y.R.; Zhang, H.Z.; Yang, B.; Yuan, L.; Shen, X.; Hu, X. Hybrid Sn-Co binary oxide nanosheets grown on carbon paper as the supercapacitor electrode materials. J. Alloys Compd. 2020, 814, 152199. [CrossRef]

71. Ramasamy, K.; Gupta, R.K.; Palchoudhury, S.; Ivanov, S.; Gupta, A. Layer-Structured Copper Antimony Chalcogenides $\left(\mathrm{CuSbSe}_{\mathrm{x}} \mathrm{S}_{2-\mathrm{x}}\right)$ : Stable Electrode Materials for Supercapacitors. Chem. Mater. 2015, 27, 379-386. [CrossRef] 
72. Lin, Y.; Dong, J.J.; Zong, H.W.; Yang, H. Synthesis and Electrochemical Properties of Coral-Like $\mathrm{LiFePO}_{4}$ Particles as Supercapacitor Electrodes. J. Electrochem. Soc. 2017, 164, A3876-A3882. [CrossRef]

73. Priyadharsini, N.; Surendran, S.; Selvan, R.K. Synthesis and electrochemical performances of $\gamma-\mathrm{KCoPO}_{4}$ nanocrystals as a promising electrode for aqueous supercapatteries. Chemelectrochem 2019, 6, 369-377. [CrossRef]

74. Elkholy, A.E.; Heakal, F.E.-T.; Allam, N.K. A facile electrosynthesis approach of amorphous Mn-Co-Fe ternary hydroxides as binder-free active electrode materials for highperformance supercapacitors. Electrochim. Acta 2019, 296, 59-68. [CrossRef]

75. Mao, X.Q.; Zou, Y.J.; Liang, J.; Xiang, C.; Chu, H.; Yan, E.; Zhang, H.; Xu, F.; Hu, X.; Sun, L. Facile synthesis of hierarchical Co-Mo-O-S porous microspheres for high performance supercapacitors. Ceram. Int. 2020, 46, 1448-1456. [CrossRef]

76. Lin, C.; Ritter, J.A.; Popov, B.N. Development of carbon-metal oxide supercapacitors from Sol-Gel derived carbon-ruthenium xerogels. J. Electrochem. Soc. 1999, 146, 3155-3160. [CrossRef]

77. Sun, Z.Y.; Liu, Z.M.; Han, B.X.; Miao, S.; Du, J.; Miao, Z. Microstructural and electrochemical characterization of RuO $2 / \mathrm{CNT}^{2}$ composites synthesized in supercritical diethyl amine. Carbon 2005, 44, 888-893. [CrossRef]

78. Min, M.K.; Machida, K.; Jang, J.H.; Naoi, K. Hydrous $\mathrm{RuO}_{2}$ / carbon black nanocomposites with 3D porous structure by novel incipient wetness method for supercapacitors. J. Electrochem. Soc. 2006, 153, A334-A338. [CrossRef]

79. Lee, Y.J.; Jung, J.C.; Park, S.; Seo, J.G.; Baeck, S.H.; Yoon, J.R.; Yi, J.; Song, I.K. Preparation and characterization of metal-doped carbon aerogel for supercapacitor. Curr. Appl. Phys. 2009, 10, 947-951. [CrossRef]

80. Sugimoto, W.; Ohnuma, T.; Murakami, Y.; Takasu, Y. Molybdenum oxide/carbon composite electrodes as electrochemical supercapacitors. Electrochem. Solid State Lett. 2001, 4, A145-A147. [CrossRef]

81. Miura, N.; Oonishi, S.; Prasad, K.R. Indium tin oxide/carbon composite electrode material for electrochemical supercapacitors. Electrochem. Solid State Lett. 2004, 7, A247-A249. [CrossRef]

82. Wang, Y.H.; Wang, C.C.; Cheng, W.Y.; Lu, S.-Y. Dispersing $\mathrm{WO}_{3}$ in carbon aerogel makes an outstanding supercapacitor electrode material. Carbon 2014, 69, 287-293. [CrossRef]

83. Yan, J.; Fan, Z.J.; Wei, T.; Qian, W.; Zhang, M.; Wei, F. Fast and reversible surface redox reaction of graphene-MnO ${ }_{2}$ composites as supercapacitor electrodes. Carbon 2010, 48, 3825-3833. [CrossRef]

84. Sethuraman, B.; Purushothaman, K.K.; Muralidharan, G. Synthesis of mesh-like $\mathrm{Fe}_{2} \mathrm{O}_{3} / \mathrm{C}$ nanocomposite via greener route for high performance supercapacitors. RSC Adv. 2014, 4, 4631-4637. [CrossRef]

85. Prasad, K.P.S.; Dhawale, D.S.; Sivakumar, T.; Aldeyab, S.S.; Zaidi, J.S.; Ariga, K.; Vinu, A. Fabrication and textural characterization of nanoporous carbon electrodes embedded with $\mathrm{CuO}$ nanoparticles for supercapacitors. Sci. Technol. Adv. Mater. 2011, 12, 044602. [CrossRef] [PubMed]

86. Liang, Y.Y.; Cao, L.; Kong, L.B.; Li, H.L. Synthesis of $\mathrm{Co}(\mathrm{OH})_{2} /$ USY composite and its application for electrochemical supercapacitors. J. Power Sources 2004, 136, 197-200. [CrossRef]

87. Lin, P.; She, Q.J.; Hong, B.L.; Liu, X.; Shi, Y.; Shi, Z.; Zhang, M.; Dong, Q. The nickel oxide/CNT composites with high capacitance for supercapacitor. J. Electrochem. Soc. 2010, 157, A818-A823. [CrossRef]

88. Dong, X.C.; Xu, H.; Wang, X.W.; Huang, Y.; Chan-Park, M.B.; Zhang, H.; Wang, L.H.; Huang, W.; Chen, P. 3D graphene-cobalt oxide electrode for high-performance supercapacitor and enzymeless glucose detection. ACS Nano 2012, 6, 3206-3213. [CrossRef] [PubMed]

89. Mo, Z.L.; Liu, P.W.; Guo, R.B.; Deng, Z.; Zhao, Y.; Sun, Y. Graphene sheets $/ \mathrm{Ag}_{2} \mathrm{~S}$ nanocomposites: Synthesis and their application in supercapacitor materials. Mater. Lett. 2012, 68, 416-418. [CrossRef]

90. Dai, C.S.; Chien, P.Y.; Lin, J.Y.; Chou, S.-W.; Wu, W.-K.; Li, P.-H.; Wu, K.-K.; Lin, T.-W. Hierarchically structured Ni $\mathrm{S}_{2} / \mathrm{carbon}$ nanotube composites as high performance cathode materials for asymmetric supercapacitors. ACS Appl. Mater. Interfaces 2013, 5, 12168-12174. [CrossRef] [PubMed]

91. Yang, S.; Wu, X.; Chen, C.; Dong, H.; Hu, W.; Wang, X. Spherical $\alpha-\mathrm{Ni}(\mathrm{OH})_{2}$ nanoarchitecture grown on graphene as advanced electrochemical pseudocapacitor materials. Chem. Commun. 2012, 48, 2773-2775. [CrossRef]

92. Liu, Y.S.; Zhitomirsky, I. Electrochemical supercapacitor based on multiferroic $\mathrm{BiMn}_{2} \mathrm{O}_{5}$. J. Power Sources 2015, $284,377-382$. [CrossRef]

93. Dong, X.Y.; Wang, L.; Wang, D.; Li, C.; Jin, J. Layer-by-layer engineered Co-Al hydroxide nanosheets/graphene multilayer films as flexible electrode for supercapacitor. Langmuir 2012, 28, 293-298. [CrossRef]

94. Xia, X.F.; Lei, W.; Hao, Q.L.; Wang, W.; Wang, X. One-step synthesis of $\mathrm{CoMoO}_{4}$ /graphene composites with enhanced electrochemical properties for supercapacitors. Electrochim. Acta 2013, 99, 253-261. [CrossRef]

95. Giri, S.; Ghosh, D.; Das, C.K. One Pot synthesis of Ilmenite-Type $\mathrm{NiMnO}_{3}$ / "Nitrogen-Doped" graphene nanocomposite as next generation supercapacitors. Dalton Trans. 2013, 42, 14361-14364. [CrossRef]

96. Grace, A.N.; Ramachandran, R.; Vinoba, M.; Choi, S.; Chu, D.; Yoon, Y.; Nam, S.; Jeong, S. Facile synthesis and electrochemical properties of $\mathrm{Co}_{3} \mathrm{~S}_{4}$-Nitrogen-doped graphene nanocomposites for supercapacitor applications. Electroanalysis 2014, 26, 199-208. [CrossRef]

97. Zhao, S.L.; Tao, K.Y.; Gong, Y. Co-Incorporated $\mathrm{NiV}_{2} \mathrm{O}_{6} / \mathrm{Ni}\left(\mathrm{HCO}_{3}\right)_{2}$ nanoflake arrays grown on nickel foam as a high-performance supercapacitor electrode. Dalton Trans. 2019, 48, 5315-5326. [CrossRef]

98. Kim, H.; Popov, B.N. Synthesis and Characterization of $\mathrm{MnO}_{2}$-Based Mixed Oxides as Supercapacitors. J. Electrochem. Soc. 2003, 150, D56-D62. [CrossRef] 
99. Zhong, J.H.; Wang, A.L.; Li, G.R.; Wang, J.; Ou, Y.; Tong, Y.X. $\mathrm{Co}_{3} \mathrm{O}_{4} / \mathrm{Ni}(\mathrm{OH})_{2}$ composite mesoporous nanosheet networks as a promising electrode for supercapacitor applications. J. Mater. Chem. 2012, 22, 5656-5665. [CrossRef]

100. Zhang, P.; Zhang, X.Q.; Li, G.Y. Fabrication of rod-like $\mathrm{NiMoO}_{4} / \mathrm{CoMoO}_{4}$ for application in asymmetric supercapacitors. Ionics 2020, 26, 393-401. [CrossRef]

101. Zhang, X.Y.; Li, Z.; Yu, Z.Y.; Wei, L.; Guo, X. Mesoporous $\mathrm{NiMoO}_{4}$ microspheres decorated by Ag quantum dots as cathode material for asymmetric supercapacitors: Enhanced interfacial conductivity and capacitive storage. Appl. Surf. Sci. 2020, 505, 144513. [CrossRef]

102. Zheng, Y.Y.; Xu, J.; Yang, X.S.; Zhang, Y.; Shang, Y.; Hu, X. Decoration $\mathrm{NiCo}_{2} \mathrm{~S}_{4}$ nanoflakes onto Ppy nanotubes as core-shell heterostructure material for high-performance asymmetric supercapacitor. Chem. Eng. J. 2018, 333, 111-121. [CrossRef]

103. Zhang, J.L.; Fu, J.C.; Zhang, J.W.; Ma, H.; He, Y.; Li, F.; Xie, E.; Xue, D.; Zhang, H.; Peng, Y. Co@Co ${ }_{3} \mathrm{O}_{4}$ core-shell three-dimensional nano-network for high-performance electrochemical energy storage. Small 2014, 10, 2618-2624. [CrossRef] [PubMed]

104. Ma, F.; Dai, X.Q.; Jin, J.; Tie, N.; Dai, Y. Hierarchical core-shell hollow CoMoS ${ }_{4}$ Ni-Co-S nanotubes hybrid arrays as advanced electrode material for supercapacitors. Electrochim. Acta 2020, 331, 135459. [CrossRef]

105. Rowlands, S.E.; Latham, R.J.; Schlindwein, W.S. Supercapacitor devices using porous silicon electrodes. Ionics 1999, 5, 144-149. [CrossRef]

106. Dai, P.C.; Zhang, S.; Liu, H.J.; Yan, L.; Gu, X.; Li, L.; Liu, D.D.; Zhao, X.B. Cotton fabrics-derived flexible nitrogen-doped activated carbon cloth for high-performance supercapacitors in organic electrolyte. Electrochim. Acta 2020, 354, 136717. [CrossRef]

107. Liu, T.W.; Zheng, Y.W.; Zhao, W.; Cui, L.; Liu, J. Uniform generation of $\mathrm{NiCo}_{2} \mathrm{~S}_{4}$ with $3 \mathrm{D}$ honeycomb-like network structure on carbon cloth as advanced electrode materials for flexible supercapacitors. J. Colloid Interface Sci. 2019, 556, 743-752. [CrossRef] [PubMed]

108. Zhao, W.; Zheng, Y.W.; Cui, L.; Jia, D.; Zheng, R.; Barrow, C.; Yang, W.; Liu, J. MOF derived Ni-Co-S nanosheets on electrochemically activated carbon cloth via an etching/ion exchange method for wearable hybrid supercapacitors. Chem. Eng. J. 2019, 371, 461-469. [CrossRef]

109. Chen, D.; Zhou, S.; Quan, H.Y.; Zou, R.; Gao, W.; Luo, X.; Guo, L. Tetsubo-like $\alpha-\mathrm{Fe}_{2} \mathrm{O}_{3} / \mathrm{C}$ nanoarrays on carbon cloth as negative electrode for high-performance asymmetric supercapacitors. Chem. Eng. J. 2018, 341, 102-111. [CrossRef]

110. Ding, R.; Zhang, M.; Yao, Y.H.; Gao, H. Crystalline $\mathrm{NiCo}_{2} \mathrm{~S}_{4}$ nanotube array coated with amorphous NiCox $\mathrm{S}_{\mathrm{y}}$ for supercapacitor electrodes. J. Colloid Interface Sci. 2016, 467, 140-147. [CrossRef]

111. Wang, L.B.; Yang, H.L.; Liu, X.X.; Zeng, R.; Li, M.; Huang, Y.; Hu, X. Constructing hierarchical tectorum-like $\alpha-\mathrm{Fe}_{2} \mathrm{O}_{3} / \mathrm{PPy}$ nanoarrays on carbon cloth for solid-state asymmetric supercapacitors. Angew. Chem. Int. Ed. 2017, 56, 1105-1110. [CrossRef]

112. Li, J.X.; Zhao, J.W.; Qin, L.R.; Zhang, Q.; Tang, X.; Xu, Y. Hierarchical $\mathrm{Co}(\mathrm{OH})_{2} @ \mathrm{NiMoS}_{4}$ nanocomposite on carbon cloth as electrode for high-performance asymmetric supercapacitors. RSC Adv. 2020, 10, 22606-22615. [CrossRef]

113. Ma, J.X.; Li, J.; Guo, R.; Xu, H.; Shi, F.; Dang, L.; Liu, Z.; Sun, J.; Lei, Z. Direct growth of flake-like metal-organic framework on textile carbon cloth as high-performance supercapacitor electrode. J. Power Sources 2019, 428, 124-130. [CrossRef]

114. Liu, J.L.; Wang, Q.; Liu, P. Poly (1,5-diaminoanthraquinone) coated carbon cloth composites as flexible electrode with extraordinary cycling stability for symmetric solid-state supercapacitors. J. Colloid Interface Sci. 2019, 546, 60-69. [CrossRef]

115. Zhang, R.P.; Liu, J.; Guo, H.G.; Tong, X. Hierarchically porous nickel oxide nanoflake arrays grown on carbon cloth by chemical bath deposition as superior flexible electrode for supercapacitors. Mater. Lett. 2014, 136, 198-201. [CrossRef]

116. Zhao, N.; Fan, H.Q.; Zhang, M.C.; Ma, J.W.; Du, Z.; Yan, B.; Li, H.; Jiang, X. Simple electrodeposition of MoO$_{3}$ film on carbon cloth for high-performance aqueous symmetric supercapacitors. Chem. Eng. J. 2020, 390, 124477. [CrossRef]

117. Javed, M.S.; Dai, S.; Wang, M.J.; Guo, D.; Chen, L.; Wang, X.; Hu, C.; Xi, Y. High performance solid state flexible supercapacitor based on molybdenum sulfide hierarchical nanospheres. J. Power Sources 2015, 285, 63-69. [CrossRef]

118. Debasis, G.S.; Manas, M.; Kumar, D.C. Solid State Flexible Asymmetric Supercapacitor Based on Carbon Fiber Supported Hierarchical $\mathrm{Co}(\mathrm{OH})_{\mathrm{x}} \mathrm{CO}_{3}$ and $\mathrm{Ni}(\mathrm{OH})_{2}$. Langmuir 2015, 31, 7835-7843.

119. Lin, J.H.; Wang, Y.H.; Zheng, X.; Liang, H.; Jia, H.; Cao, J.; Tu, J.; Fei, W.; Feng, J. P-Doped $\mathrm{NiCo}_{2} \mathrm{~S}_{4}$ nanotubes as battery-type electrodes for high-performance asymmetric supercapacitors. Dalton Trans. 2018, 47, 8771-8778. [CrossRef]

120. Liang, H.Y.; Lin, J.H.; Jia, H.; Chen, J.; Cao, J.; Lin, T.; Fei, W.; Feng, J. Hierarchical NiCo-LDH/NiCoP@NiMn-LDH hybrid electrodes on carbon cloth for excellent supercapacitors. J. Mater. Chem. A 2018, 6, 15040-15046. [CrossRef]

121. Horng, Y.Y.; Lu, Y.C.; Hsu, Y.K.; Chen, C.C.; Chen, L.C.; Chen, K.H. Flexible supercapacitor based on polyaniline nanowires/carbon cloth with both high gravimetric and area-normalized capacitance. J. Power Sources 2010, 195, 44184422. [CrossRef]

122. Sekhar, S.C.; Nagaraju, G.; Yu, J.S. Conductive silver nanowires-fenced carbon cloth fibers-supported layered double hydroxide nanosheets as a flexible and binder-free electrode for high-performance asymmetric supercapacitors. Nano Energy 2017, 36, 58-67. [CrossRef]

123. Shakir, I.; Shahid, M.; Rana, U.A.; Al Nashef, I.M.; Hussain, R. Nickel-cobalt layered double hydroxide anchored zinc oxide nanowires grown on carbon fiber cloth for high-performance flexible pseudocapacitive energy storage devices. Electrochim. Acta 2014, 129, 28-32. [CrossRef]

124. Torrinha, Á.; Morais, S. Electrochemical (bio)sensors based on carbon cloth and carbon paper: An overview. TRAC-Trends Anal. Chem. 2021, 142, 116324. [CrossRef] 
125. Mishra, A.; Shetti, N.P.; Basu, S.; Reddy, K.R.; Aminabhavi, T.M. Carbon Cloth-based Hybrid Materials as Flexible Electrochemical Supercapacitors. ChemElectroChem 2019, 6, 5771-5786. [CrossRef]

126. Wang, Q.; Wu, M.; Meng, S.; Zang, Z.; Si, W.; Huang, W.; Dong, X. Hydrazine Sensor Based on $\mathrm{Co}_{3} \mathrm{O}_{4} / \mathrm{rGO} / \mathrm{Carbon} \mathrm{Cloth}$ Electrochemical Electrode. Adv. Mater. Interface 2016, 3, 1500691. [CrossRef]

127. Zhang, J.; Liu, P.; Jin, C.; Jin, L.; Bian, S.; Zhu, Q.; Wang, B. Flexible three-dimensional carbon cloth $/$ carbon fibers $/ \mathrm{NiCo}_{2} \mathrm{O}_{4}$ composite electrode materials for high-performance all-solid-state electrochemical capacitors. Electrochim. Acta 2017, 265, 90-99. [CrossRef] 